\title{
Experimental determination and predictive modelling of the mutual diffusion coefficients of water and ionic liquid 1-(2-hydroxyethyl)-3- methylimidazolium tetrafluoroborate
}

\author{
R. Rives ${ }^{\mathrm{a}}$, A. Mialdun ${ }^{\mathrm{b}}$, V. Yasnou ${ }^{\mathrm{b}}$, V. Shevtsova ${ }^{\mathrm{b}}$, A. Coronas $^{\mathrm{a}}$ \\ aUniversitat Rovira i Virgili, Mechanical Engineering Department, Crever, Av. Països \\ Catalans 26, 43007, Tarragona, Spain \\ bUniversité libre de Bruxelles, Microgravity Research Center, Av. F.D. Roosevelt 50, 1050 \\ Brussels, Belgium \\ ‘amialdun@ulb.ac.be
}

Keywords:

Diffusion coefficient, Optical digital interferometry, Predictive diffusion models, Ionic liquid.

\begin{abstract}
Mutual diffusion coefficients of a binary mixture of water + ionic liquid 1-(2-hydroxyethyl)-3methylimidazolium tetrafluoroborate have been investigated by means of experimental and predictive approaches. Optical digital interferometry has been employed to determine the mutual diffusion coefficients over the full ionic liquid mass fraction range and at four temperatures between 298.15-313.15 K. Moreover, the prediction ability of four different models has been tested. The influence of using different excess Gibbs energy $\left(g^{\mathrm{E}}\right)$ models to calculate the relevant thermodynamics properties was also addressed. The mutual diffusion coefficient has a strong dependence on concentration and varies from $2 \times 10^{-10}$ to $1.27 \times 10^{-9}$ $\mathrm{m}^{2} \mathrm{~s}^{-1}$. The concentration and temperature dependencies of the present experimental diffusion coefficients were correlated using empirical and predictive equations. The use of binary interaction parameter regressed from diffusion data instead of vapor-liquid equilibria improves considerably the prediction ability of the employed predictive models. Among the studied predictive models, the modified group contribution model provides the best results with an absolute relative deviation of $2.1 \%$. Throughout the paper, thermodynamic and kinetic behavior of the mixture are analyzed for establishing an appropriate criterion for the screening of ionic liquids as working fluids for absorption refrigeration systems.
\end{abstract}

\section{Introduction}

Ionic liquids (ILS) are a class of molten salts that have unique physical and chemical properties such as negligible vapor pressure, good solubility to many organic or inorganic chemicals, and a wide range in liquid state from room temperature to about $300{ }^{\circ} \mathrm{C}$ [1]. Because of these properties, ILs have been adopted as new "green solvents" and extensively studied for separation processes and carbon capture [2]. Moreover, the properties of ionic liquids can be adjusted to meet the specific requirements of a given application through the proper selection of cation and anion [3]. Therefore, their use has been proposed in a huge variety of applications, such as absorption refrigeration systems [4]; as electrolyte and electrode materials for batteries [5]; optical applications like high refractive index glass and crystal analysis [6], in space propulsion [7], etc.

One of the applications where the ionic liquids have received great attention is absorption refrigeration systems and heat pumps [7]. These systems employ a mixture of two fluids (namely, refrigerant and absorbent) with different volatilities as working fluids. Absorption systems using natural refrigerants (mainly $\mathrm{H}_{2} \mathrm{O}, \mathrm{NH}_{3}, \mathrm{CO}_{2}$ ) and ILs as absorbent have been extensively studied [8-12]. Considering that there is 
an infinity of anion and cation combinations for potential ionic liquids, the screening of the ILs as working fluids is critically important.

One common thermodynamic criterion for the screening of ILs as working fluids is based on the minimum value of the excess Gibbs energy ( $g^{E}-x$ relationship). A lower value of the excess Gibbs energy means that there is a high chemical affinity of IL for a given refrigerant. Cera-Manjarres et al. [8] used the $\mathrm{g}^{\mathrm{E}}-\mathrm{x}$ relationship and the deviation from Raoult's law for selecting the most suitable IL absorbent for ammonia. Among the investigated ILs, ionic liquid 1- (2-hydroxyethyl) -3-methylimidazolium tetrafluoroborate $[\mathrm{EtOHmim}]\left[\mathrm{BF}_{4}\right]$ was selected as the most suitable.

Despite the importance of the criterion of excess Gibbs energy, in practice, the performance of absorption systems is affected not only by thermodynamics but also by kinetics. The kinetics of the absorption process is dominated by transport properties, such as the mutual diffusion coefficient and viscosity of the working fluids. These properties have a great influence on the heat and mass transfer rates in the absorption cycle and, therefore, on the performance of the system. For example, a low refrigerant diffusivity in the ionic liquid requires such a great mass transfer area, that makes the system technically infeasible. In addition, the high viscosity of ILs have been identified as the main shortcoming for their applications in absorption systems. Therefore, some authors [13-15] have proposed the use of IL as additives in conventional absorbent media, such as water. In this way, instead of the binary system refrigerant + absorbent, the working fluids would be the ternary system refrigerant (such as ammonia) + absorbent (other than ILs, such as water) + ILs (such as [EtOHmim] $\left[\mathrm{BF}_{4}\right]$ ) as additives. The thermodynamic and kinetic analysis of ternary mixtures is much more complex than in the binary case, so it is important to know binary limits first.

The present study is focused on the mutual diffusion coefficient because this property is essential for the proper design of equipment in absorption systems and in any industrial application involving mass transfer processes. Very few studies have been published concerning the diffusion of ionic liquids in common solvents of industrial and environmental importance $[2,16]$. Accordingly, a binary mixture of water and ionic liquid [EtOHmim] $\left[\mathrm{BF}_{4}\right]$, which is a promising working fluid for absorption systems, was chosen as the object of the investigation.

Herein, the study combines experimental work, and predictive models to determine the mutual diffusion coefficients of the binary mixture $\mathrm{H}_{2} \mathrm{O}+[\mathrm{EtOHmim}]\left[\mathrm{BF}_{4}\right]$.

The experimental determination of mutual diffusion coefficients relies on the use of precise measurement techniques and adequate processing methods. In this work, the diffusion process in a binary mixture has been investigated by interferometric probing, in particular, optical digital interferometry (ODI). This method has been successfully used to determine diffusion and thermodiffusion coefficients in binary and ternary classical liquid systems [17-19]. The unique feature of the ODI method is that it traces the transient path of the system over the entire 2D cell cross-section throughout the whole diffusion process [20].

There is a growing demand for accurate diffusion properties, which experimental measurements alone are not able to satisfy. Considering the huge family of ionic liquids, the need to predict the mutual diffusion coefficient becomes evident. 
However, predictive correlations do not often give satisfactory results, and there is a great interest in improving their performance. The development of reliable theoretical approaches is limited by the complexity of solute-solvent interactions and enormous molecular structures of some media, such as ionic liquids. Moreover, most of the developed models consider the mutual diffusion coefficient as the product of a compositional average of limiting diffusion coefficients (kinetics contribution) and a thermodynamic factor (thermodynamic contribution) which represents the non-ideality of the mixture [21-23].

Therefore, one of the aims of this work is to complement the experimental results over the entire composition range by comparing with four predictive approaches: Vignes [21], UNIDIF [24], Modified Group Contribution [22], and Cluster diffusion [23]. To the best of our knowledge, it is the first time that some of these models have been used to predict the diffusion coefficient in binary mixtures with ionic liquids.

This article is organized as follows. First, a section where the studied predictive models are presented. Second, a section where the experimental approach is described in detail. Third, the behavior of the relevant thermodynamic properties related to diffusion is discussed, and the experimental and predictive results are presented, analyzed and compared with each other. Finally, conclusions are drawn.

\section{Predictive approach}

As mentioned before, many predictive models have been developed for mutual diffusion coefficients in binary liquid mixtures. Predicted diffusion coefficients reveal better agreement with experimental data for ideal or nearly ideal mixtures, meanwhile errors can be quite large for non-ideal and non-associated solutions [25].

Herein, for the predictive models based on the thermodynamic factor, the influence of using different $\mathrm{g}^{\mathrm{E}}$ models is analyzed and quantified. The use of $\mathrm{g}^{\mathrm{E}}$ models to calculate the thermodynamic factor, and hence to predict the mutual diffusion coefficient, allows generalizing the criterion of excess Gibbs energy for the screening of ILs working fluids in absorption systems and incorporates kinetic considerations. In addition, the use of binary interaction parameters from vapor-liquid equilibrium (VLE) data and from experimental diffusion data is also considered. The analysis of optimal binary interaction parameters allows evaluating the suitability of the vapor-liquid equilibrium (VLE) data to describe the kinetics of this mixture.

In this section, four different predictive models for the mutual diffusion coefficient, namely Vignes, UNIDIF, Modified Group Contribution, and Cluster diffusion, are briefly described. This section also discusses how to use these models for the mixture under study.

\subsection{Classical models. Vignes correlation.}

In binary liquid mixtures, the mutual diffusion coefficient, $D_{12}$, is described by several correlations as a function of composition and some limiting diffusion coefficients. One of the first correlations, which is still used quite frequently, was proposed by Darken [26] as: 
where $x_{1}, x_{2}$ are the mole fractions of componets 1 and 2, respectively; $\gamma_{1}$ is the activity coeficient of component 1 ; and $D_{1}^{*}, D_{2}^{*}$ are the tracer diffusion coefficient of components 1 and 2, respectively. Therefore, to use the Darken correlation, one has to know the tracer diffusion coefficient of the components, which is not always available in the literature. It is important to note that in this correlation the mutual diffusion coefficient is expressed by a kinetic contribution $\left(x_{2} D_{1}^{*}+x_{1} D_{2}^{*}\right)$ and a thermodynamic contribution $\left[1+x_{1} \frac{d l n \gamma_{1}}{d x_{1}}\right]$. Therefore, this last term is called the thermodynamic factor and represents the deviation from ideality of a mixture.

Later, Vignes [21] conveniently replaced the tracer diffusion coefficients in the Darken equation by the limiting diffusion coefficients at infinite dilution. Vignes correlation is expressed as the product of a geometric average of the composition and a thermodynamic factor as follows:

$$
D_{12}=\left(\left(D_{1}^{\infty}\right)^{x_{2}}\left(D_{2}^{\infty}\right)^{x_{1}}\right)\left[1+x_{1} \frac{d \ln \gamma_{1}}{d x_{1}}\right]
$$

where $D_{1}^{\infty}, D_{2}^{\infty}$ are the limiting diffusion coefficients at infinite dilution of components 1 and 2, respectively. There are several avalaible correlations for calculation of diffusion coefficients at infinite dilution with reasonable success. In the Vignes corelation, $\Gamma=1+x_{1} \frac{d \ln \gamma_{1}}{d x_{1}}$ is the thermodynamic factor which can be obtained from an $\mathrm{g}^{\mathrm{E}}$ model as described below. It is important to note that the thermodynamic factor can be calculated from the activities and mole fractions of either component 1 or component 2 according to the Gibbs-Duhem equation [25].

The correlations shown above, Darken and Vignes ones, are both classical interpolation methods that can predict the diffusion coefficient in ideal or nearly ideal solutions quite well. However, in non-ideal solutions, the prediction of the mutual diffusion coefficient is not very accurate, and deviation from experimental data could be large. Despite this, these correlations have been thoroughly tested and form the basis of more sophisticated diffusion models. Thus, in this work we analyzed the performance of one of them for the mixture of interest $\left(\mathrm{H}_{2} \mathrm{O}+[\mathrm{EtOHmim}][\mathrm{BF} 4]\right)$. Between the two correlations, we have selected the Vignes correlation because no tracer diffusion coefficients are necessary as in the case of Darken correlation.

\subsection{UNIDIF model}

In another approach, Hsu and Chen [24] developed a correlation for the mutual diffusion coefficient of binary liquid mixtures using statistical thermodynamics and the absolute reaction rate theory. The developed correlation does not involve the use of a thermodynamic factor, instead it expresses the diffusion coefficient as a reference term and an excess term. The reference term is based on a mole fraction average of the logarithm of the limiting diffusion coefficients. Meanwhile, the excess part of the diffusion coefficient is expressed as UNIQUAC equation, which comprises two parts due to the combinatorial and residual contributions. The combinatorial part depends on the molecular sizes and shapes. The residual part includes two binary interaction parameters, which can be obtained from experimental data. 
In this model, the mutual diffusion coefficient of a binary mixture can be expressed as:

4

$$
\begin{aligned}
\ln D_{12}= & x_{2} \ln D_{1}^{\infty}+x_{1} \ln D_{2}^{\infty}+2\left(x_{1} \ln \frac{x_{1}}{\phi_{1}}+x_{2} \ln \frac{x_{2}}{\phi_{2}}\right)+ \\
& +2 x_{1} x_{2}\left[\frac{\phi_{1}}{x_{1}}\left(1-\frac{\lambda_{1}}{\lambda_{2}}\right)-\frac{\phi_{2}}{x_{2}}\left(1-\frac{\lambda_{2}}{\lambda_{1}}\right)\right]+ \\
+ & x_{2} q_{1}\left[\left(1-\theta_{21}^{2}\right) \ln \tau_{21}+\left(1-\theta_{22}^{2}\right) \tau_{12} \ln \tau_{12}\right]+ \\
+ & x_{1} q_{2}\left[\left(1-\theta_{12}^{2}\right) \ln \tau_{12}+\left(1-\theta_{11}^{2}\right) \tau_{21} \ln \tau_{21}\right]
\end{aligned}
$$

Here, $\theta_{j i}$ (for $j$ or $i=1$ or 2 ) is the local composition parameter, and it is defined by Abrams and Prausnitz [27] as:

$$
\theta_{j i}=\frac{\theta_{j} \tau_{j i}}{\sum_{l}^{2} \theta_{l} \tau_{l i}}
$$

where $\theta_{j}$ is the average fraction of the surface area $q$ of the component $j$ :

$$
\theta_{j}=\frac{x_{j} q_{j}}{\sum_{l}^{2} x_{l} q_{l}}
$$

$\tau_{j i}$ is the binary interaction parameter defined as:

$$
\tau_{j i}=\exp \left(-\frac{a_{j i}}{T}\right)
$$

where $T$ is the temperature and $a_{j i}$ are the adjustable parameters.

Finally, $\phi_{i}$ (for $i=1$ or 2 ) is defined as:

$$
\phi_{i}=\frac{x_{i} \lambda_{i}}{\sum_{l}^{2} x_{l} \lambda_{l}}
$$

where $\lambda_{i}=\left(r_{i}\right)^{\frac{1}{3}}$, and $r_{i}$ are the UNIFAC volume parameters.

Hsu and Chen determined values of the interaction parameters for 49 binary systems. They evaluated the performance of their correlation by testing 1042 experimental points and found an average error of $2.3 \%$. They compared their results with those from modified Darken and Vignes correlations for the same systems and concluded that the developed correlation gave superior results.

In order to use this method, ionic liquid [EtOHmim][BF 3 has to be segmented into groups. We have divided ionic liquid into four groups, one $\mathrm{OH}$ group, two $\mathrm{CH}_{2}$ groups, and one [mim] $\left[\mathrm{BF}_{4}\right]$ group as it is shown in Figure 1. Here, we followed the method proposed by Lei et al. [28]. Table 1 shows the volume parameter $R_{j}$ and surface parameter $Q_{j}$ per group used in this study. 


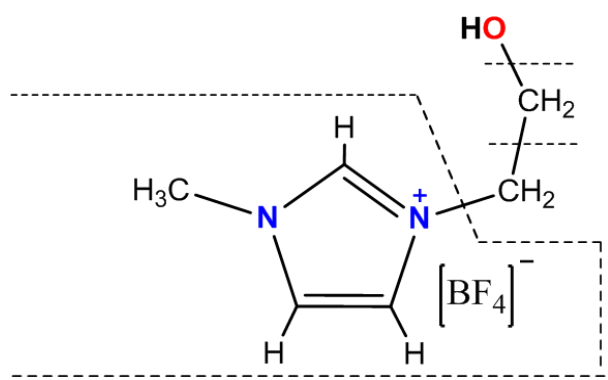

Figure 1. Group segmentation of ionic liquid [EtOHmim][BF 4 .

Table 1. Group parameters of volume $\mathrm{Rj}$ and surface area Qj for the binary mixture $\mathrm{H}_{2} \mathrm{O}+$ [EtOHmim] $\left[\mathrm{BF}_{4}\right]$.

\begin{tabular}{|c|c|c|}
\hline Group & Volume $\left(\boldsymbol{R}_{\boldsymbol{j}}\right)$ & Surface Area $\left(\boldsymbol{Q}_{\boldsymbol{j}}\right)$ \\
\hline $\mathrm{H}_{2} \mathrm{O}$ & 0.9200 & 1.4000 \\
\hline $\mathrm{OH}$ & 1.0000 & 1.2000 \\
\hline $\mathrm{CH}_{2}$ & 0.6744 & 0.5400 \\
\hline$[\mathrm{mim}][\mathrm{BF} 4]$ & 6.5669 & 4.0050 \\
\hline
\end{tabular}

Finally, surface and volume parameters of ionic liquid $[\mathrm{EtOHmim}]\left[\mathrm{BF}_{4}\right]$ can be calculated from the group parameters as:

$$
\begin{aligned}
& q_{\text {EtOHmimBF } 4}=\sum_{j=1}^{n} v_{\text {EtOHmimBF } 4 j} Q_{j} \\
& r_{\text {EtoHmimBF4 }}=\sum_{j=1}^{n} v_{\text {EtOHmimBF } 4 j} R_{j}
\end{aligned}
$$

where $v_{\text {EtoнmimBF4j }}$ is the number of group $\mathrm{j}$ in [EtOHmim] $\left[\mathrm{BF}_{4}\right]$.

\subsection{Cluster diffusion model}

More recently, Kamgar et al.[23] developed a correlation to predict mutual diffusion coefficients in concentrated solutions. The correlation is based on the cluster diffusion theory proposed by Cussler [29]. In this theory, the diffusion is represented through the movement of entire clusters of molecules. The correlation developed by Kamgar et al. expresses the diffusion coefficient as a function of temperature $(T)$, viscosity $(\eta)$ and composition of the solution $\left(x_{1}\right.$ and $\left.x_{2}\right)$ as follows:

$$
D_{12}=\frac{k T}{2 \pi \eta(2-n) \beta r_{0}}\left(\frac{1}{1+Y^{*} \frac{1}{x_{1} x_{2}}\left(\frac{1}{\Gamma}-1\right)}\right)^{\frac{1}{3-n}}
$$

where $k$ is the Boltzmann constant, $r_{0}$ is the radius of the solute molecule, $\Gamma=1+$ $x_{1} \frac{d \ln \gamma_{1}}{d x_{1}}$ is the thermodynamic factor, and $n, \beta, Y^{*}$, are the adjustable parameters of the model, respectively. Here, the radius of the solute for the ionic liquid is unknown, so in this work, it is used as another adjustable parameter.

Kamgar et al. [23] evaluated the prediction ability of the developed expression by comparison with the experimental data of 11 binary systems from literature. Moreover, they tested three different approaches to obtain the thermodynamic factor, namely NRTL, Wilson, and numerical integration of the VLE data. They found good agreement with the experimental data for classical binary systems, with an average relative error of $4.24 \%, 3.47 \%$, and $13.99 \%$ when using NRTL, Wilson, and numerical integration, respectively. 
In order to use this diffusion model, the solution viscosity is needed. In this work, we have adopted a model to obtain the solution viscosity as follows [30]:

$$
\ln \eta=\sum_{i=1}^{N} \xi_{i} \ln \eta_{i}
$$

where:

$$
\xi_{i}=\frac{M_{i}^{c} x_{i}}{\sum_{i=1}^{N} M_{i}^{c} x_{i}}
$$

$M_{i}$ is the molecular weight of the ith species, and $c$ is an empirical parameter that enters in the pool of the adjustable parameters of the model. Pure component viscosities, $\eta_{i}$, as a function of temperature are taken from literature [31] and [32] for [EtOHmim] $\left[\mathrm{BF}_{4}\right]$ and $\mathrm{H}_{2} \mathrm{O}$, respectively.

\subsection{Modified group contribution model (MGC)}

In one of the very few available investigations applied to absorption systems, Chen et al. [22] used a modified group contribution (MGG) model to determine the mutual diffusion coefficient in a $\mathrm{H}_{2} \mathrm{O}+$ ionic liquid binary system. They studied mixtures of ionic liquid (1-methyl-3-methylimidazolium dimethylphosphate ([mmim]DMP) with water and methanol. In this model, the mutual diffusion coefficient is described as a diffusion coefficient $D_{12}$ id for an ideal solution modified by a thermodynamic correction factor $\psi$ as:

$$
D_{12}=\psi D_{12}{ }^{i d}
$$

Here the mutual diffusion coefficient for the ideal solution is written as follows:

$$
D_{12}^{i d}=D^{*} \frac{\left(x_{1} q_{2}+x_{2} q_{1}\right)^{a}}{\left(x_{1} q_{1}+x_{2} q_{2}\right)^{b}} \sqrt{T} \exp \left(\frac{\left(x_{1} E_{21}+x_{2} E_{12}\right)}{R T}-\alpha \frac{298.15\left(x_{1} r_{2}+x_{2} r_{1}\right)^{c}}{T\left(x_{1} q_{1}+x_{2} q_{2}\right)^{d}}\right)
$$

where $x_{1}$ and $x_{2}$ are mole fractions of components, $E_{12}$ and $E_{21}$ are interaction energies, $R$ is the universal gas constant, $T$ is the temperature, and $q_{1}, q_{2}, r_{1}, r_{2}$ are the surface and volume parameters defined above.

The thermodynamic correction factor used by Chen et al. is related with the thermodynamic factor by:

$$
\psi=\Gamma^{\beta}=\left[1+x_{2} \frac{d \ln \gamma_{2}}{d x_{2}}\right]^{\beta}
$$

where $x_{2}$ and $\gamma_{2}$ are the mole fraction and the activity coefficient of the small molecule component, respectively. The activity coefficients used in their work were obtained from VLE data in the literature.

In the MGC model, there are 7 adjustable parameters, $D^{*}, \alpha, \beta, a, b, c$, and $d$, used to correlate the experimental data. Chen et al. [22] found a very good agreement with the experimental data. They obtained an average absolute relative deviation of $2.05 \%$ and $1.78 \%$, for the $[\mathrm{mmim}] \mathrm{DMP} / \mathrm{H}_{2} \mathrm{O}$ and $[\mathrm{mmim}] \mathrm{DMP} / \mathrm{CH}_{3} \mathrm{OH}$ systems, respectively. 


\section{Experimental approach}

In this section, the experimental strategy employed to measure mutual diffusion coefficients in the mixture $\mathrm{H}_{2} \mathrm{O}+[\mathrm{EtOHmim}]\left[\mathrm{BF}_{4}\right]$ is described in detail.

\subsection{Materials, set-up and experimental procedure}

For the experimental measurement of the diffusion coefficient, a closed diffusion cell with interferometric probing was used. Ionic liquid [EtOHmim][BF4] (1-(2hydroxyethyl)-3-methylimidazolium tetrafluoroborate, >98\%, CAS Number: 37456483-7, Molecular Weight: 213.97) supplied by lolitec, and Water extra pure, deionized, supplied by Acros Organics were used without further purification. It is worth mentioning that the water was degassed to avoid the formation of bubbles, which can seriously affect the concentration distribution of the components inside the diffusion cell. Before starting the experiments, mixtures of $\mathrm{H}_{2} \mathrm{O}+[\mathrm{EtOHmim}]\left[\mathrm{BF}_{4}\right]$ were prepared gravimetrically with an accuracy of $\pm 0.005 \mathrm{~g}$. For each experimental run, two solutions with a small mass fraction difference of $1 \%$ were treated as one pair for the diffusion measurement at the mean composition.

The schematic design of the diffusion cell used for the experiments is shown in Figure 2. The optical path length inside the liquid bulk is $L=5.0 \mathrm{~mm}$, the total height is $H=$ $20.0 \mathrm{~mm}$, and the width of the cell is $W=5.0 \mathrm{~mm}$. The location of the diffusion cell in the experimental set-up can be appreciated in Figure 3. The experimental procedure was established as follows. At the beginning of each experimental run, the cell was filled with the heavier solution through the bottom inlet. We let the heavier solution flow into the cell for some time just to ensure that it removes any residual liquid from previous experiments. At the end of this phase, the injection of the heavier solution was stopped, and the lighter solution was injected from the top inlet until it reaches mid-height. After that both liquids are injected into the cell simultaneously by a syringe pump through the orifices in the bottom (a denser liquid) and top (a lighter liquid) walls. The quality and thickness of the interface depend on the injection flow rate. While a high injection flow rate deforms the interface, a slow filling of the cell produces a wide interface. After several tests, a flow rate equal to $2 \mathrm{ml}$ per hour was chosen for the experiments. It provides a fairly good interface sharpness, a typical example of which can be seen in the first snapshot in Figure 4.

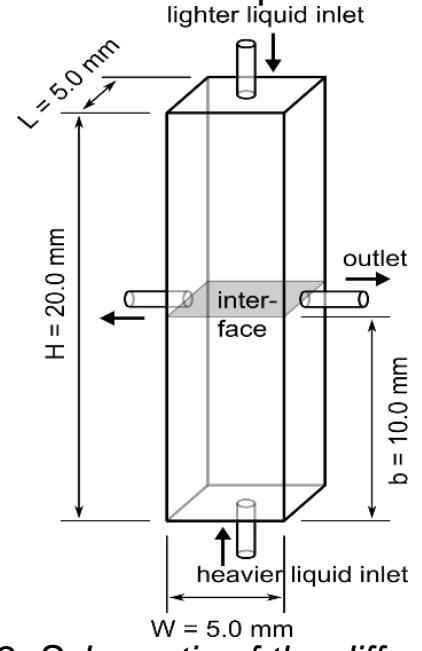

Figure 2. Schematic of the diffusion cell. 
The injection systems consist of a dual syringe pump from kdScientific, model KDS 200/200P. The diffusion cell and all optical components were maintained inside a thermally insulated box equipped with an active thermal control system. The temperature inside the box was regulated between $298.15 \mathrm{~K}$ to $313.15 \mathrm{~K}$ with residual fluctuations below $0.1 \mathrm{~K}$.

An optical arrangement based on a Mach-Zehnder interferometer was employed to sample refractive index changes inside the diffusion cell. A sketch of the experimental set-up is shown in Figure 3. The light source was an expanded and collimated laser beam with a wavelength of $\lambda=670 \mathrm{~nm}$. The resulting interferogram was recorded by a CCD camera with a $1280 \times 1024$ pixels sensor. The resolution of the imaging system was around $15.7 \mu \mathrm{m}$ per pixel. The image acquisition time step was varied from $10 \mathrm{~s}$ at the beginning of the experiment to $300 \mathrm{~s}$ at the end. Then the set of images was analyzed to extract an optical phase, from which the refractive index change profiles were obtained. Finally, mutual diffusion coefficients were evaluated by fitting an analytical solution to the obtained experimental data.

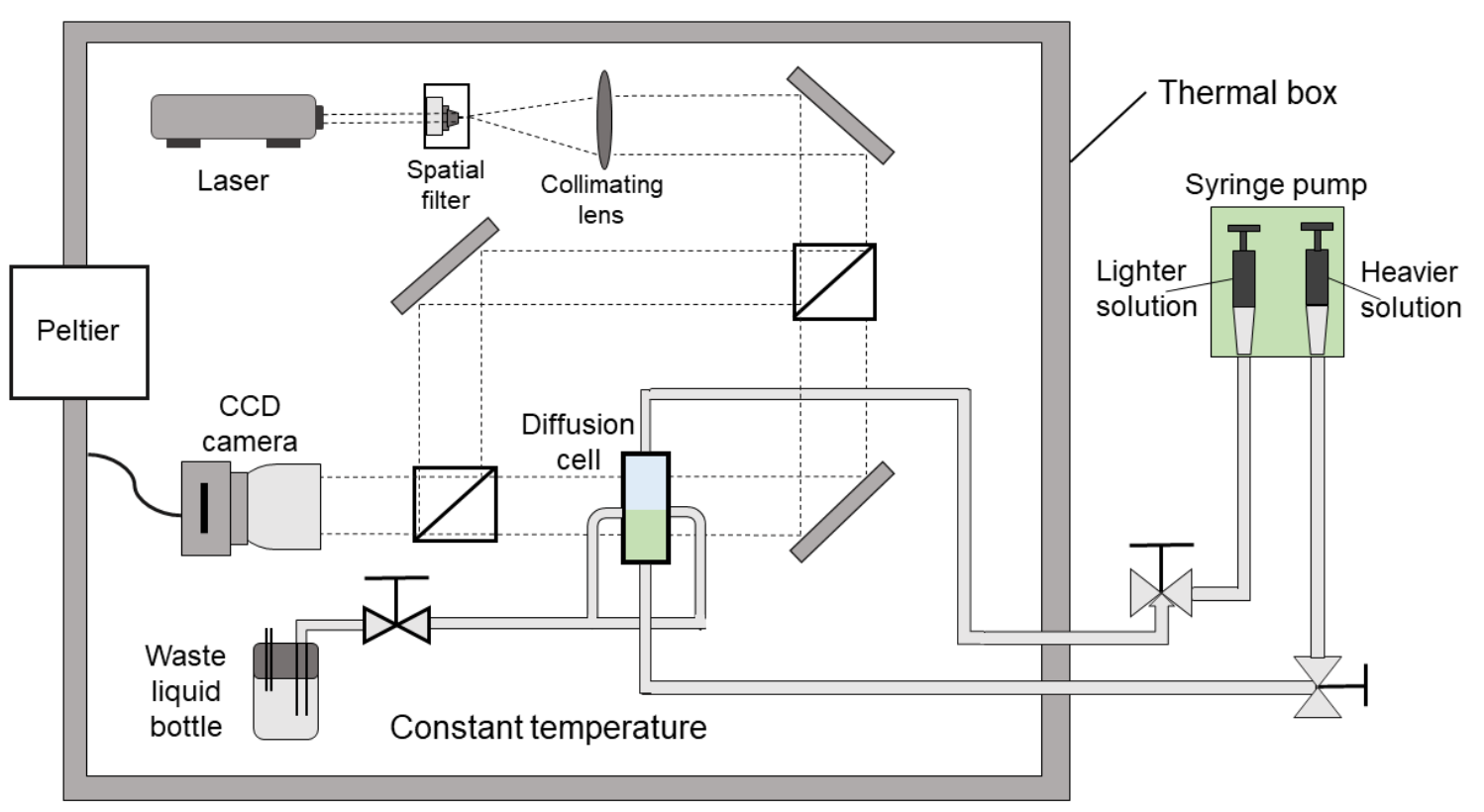

Figure 3. Schematic of the experimental set-up.

\subsection{Image processing and determination of refractive index profiles}

As mentioned above, the experimental output of the ODI method is an interferogram. Figure 4 shows original experimental interferograms, corresponding to a variation of the refractive index at different time instances. In the first picture (at the beginning of the experiment), the interface is quite sharp. In the second image (after 15 minutes), the relaxation of the interface clearly indicates that a diffusion process is taking place. Here, 2D Fourier transform technique was employed for the extraction of an optical phase from the interferograms. The image processing consists of applying the 2D Fourier transform to the interferogram, selecting one of the lateral peaks in the Fourier domain, moving the selected peak towards the origin and applying the inverse Fourier transform. More detailed descriptions are available in the literature [19]. After these steps, the results are a set of two-dimensional (2-D) phase map images wrapped into $[0,2 \pi)$ range; an example is shown in the fourth snapshot in Figure 4. 
The next step in the image processing, is the selection of two phase images, one to be processed and the other as the reference. For a proper estimation of an optical phase, a reference image is needed. This reference image will be subtracted from each of the following images to separate the value of interest. The selection of a proper reference image is a very important step because the presence of tiny refractive index imperfections can lead to data corruption. Ideally, the reference image should provide a pure background with no traces of refractive index differences in the diffusion cell, which is difficult to achieve in practice. We have thus decided to use the very last image of the experiment as the reference [20].

To minimize the computational cost, first, we made the subtraction of the reference phase image from the other phase image and only then we proceeded to perform the unwrapping. The unwrapping procedure was based on the simple pixel-to-pixel comparing principle but with a specifically oriented unwrapping path. The unwrapping was oriented from the region with the best phase quality toward the regions with the worst phase quality [33]. Wrapped and unwrapped phase maps after subtraction of the reference image are shown in the last two pictures in Figure 4-at $t=15 \mathrm{~min}$.
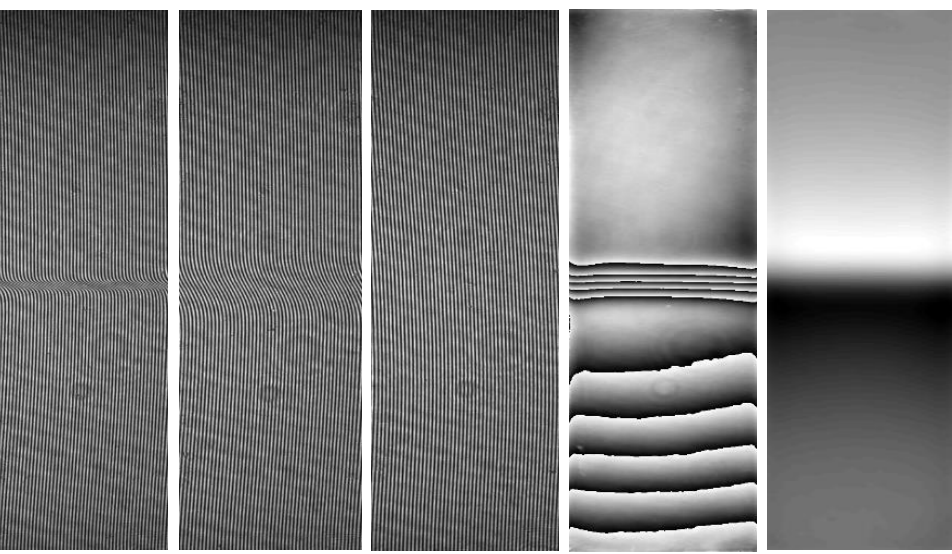

Figure 4. Transient diffusion fields: the three pictures on the left are fringe images at $t=0,15 \mathrm{~min}$ and 15 hours, respectively; the following two pictures show the wrapped and unwrapped phase at $t=15$ min after the beginning of the experiment.

Then, the 2-D unwrapped phase map $(\Delta \varphi(x, z))$ was converted into a map of refractive index $\left(\Delta n_{\text {exp }}(x, z)\right)$ by applying the relation:

$$
\Delta n_{\text {exp }}(x, z)=\frac{\lambda}{2 \pi L} \Delta \varphi(x, z)
$$

where $x, z$ are the horizontal and vertical directions, respectively; $L=5.0 \mathrm{~mm}$ is the optical path length inside the liquid bulk and $\lambda$ is the wavelength.

The ODI technique gives a unique possibility of increasing measurement accuracy by providing information about composition distribution along the whole diffusion path. It provides a two-dimensional refractive index field, although the distribution itself is almost one-dimensional. To apply 1D mathematical description of measurements, the 2-D map of the refractive index is averaged in the horizontal direction. The averaging also increases the reliability of the extracted profiles because it suppresses local noise, which is otherwise observable, without applying additional filters. 


\subsection{Working equations}

Mutual diffusion coefficients were determined by fitting an analytical solution to the experimental refractive index profiles. For this purpose, two different routes were followed. In each case, the mathematical description of the experiment is different, but both led to accurate fitting results. Therefore, the resulting mutual diffusion coefficient values using these routes did not deviate significantly from each other. In both routes, the calculated refractive index profile $\Delta n_{c a l}(z, t)$ is expressed as:

$$
\Delta n_{\text {cal }}(z, t)=\Delta n_{0} * f(z, t, D)
$$

where $\Delta n_{0}$ is the initial refractive index difference between the top and bottom solutions; and $f(z, t, D)$ is the function presenting the solution of the diffusion problem with initial and boundary conditions of the diffusion cell. This function depends on spatio-temporal variables and the unknown diffusion coefficient. The form of this function for the considered geometry and initial conditions here is shown below.

\subsubsection{Long-time model}

When the diffusion time is long, the diffusing fronts reach the ends of the cell, and the problem should be considered as occurring in a finite medium. In this case, the function $f(z, t, D)$ can be expressed as:

$$
f(z, t, D)=\frac{2}{\pi} \sum_{n=1}^{\infty} \frac{1}{n} \sin \left(\frac{n \pi b}{H}\right) \cos \left(\frac{n \pi z}{H}\right) \exp \left(-\frac{n^{2} \pi^{2}}{H^{2}} D t\right)
$$

where $b$ is the distance between the initial interface and the bottom of the diffusion cell, $H$ is the height of the cell, $D$ is the mutual diffusion coefficient and $t$ is the time.

Then, the mutual diffusion coefficient is obtained by minimization of the deviation between experimental and calculated refractive index profiles as:

$$
\Phi=\sum_{i, j}\left[\Delta n_{\text {exp }}\left(z_{i}, t_{j}+t_{0}\right)+\Delta n_{\text {cal }}\left(z_{i}, t_{\mathrm{end}}+t_{0}\right)-\Delta n_{c a l}\left(z_{i}, t_{j}+t_{0}\right)\right]^{2}
$$

Mutual diffusion coefficient and initial refractive index difference are determined as the result of minimization. Here, $t_{\text {end }}$ is the timestamp of the last image, which was taken as a reference. In this equation, $t_{0}$ is an empirical parameter introduced to consider that the initial distribution of the refractive index recorded in the experiment does not have a perfect stepwise shape at the interface, as it is assumed by the analytical solution. In other words, $t_{0}$ is the first time instance at which the theoretical profile coincides with the experimental one. Therefore, three unknown parameters were concurrently fitted: $D, \Delta n_{0}$ and $t_{0}$.

\subsubsection{Short-time model}

The other route followed to obtain the mutual diffusion coefficients considers that the diffusion fronts does not reach the top and bottom of the diffusion cell. In this case, the diffusion process can be considered as occurring in an infinite medium. In this case, function $f(z, t, D)$ in $\Delta n c a l z, t]=\Delta n_{0} * f(z, t, D)(17)$ can be expressed as: 


$$
f=\frac{1}{2}\left[\operatorname{erfc}\left(\frac{z-b}{2 \sqrt{D t}}\right)-1\right]
$$

The fitting procedure for extracting the mutual diffusion coefficients and the other fitting parameters is similar to the long-time model.

\section{Results and discussion}

In this section, the calculated thermodynamic properties, and the experimental and predictive results are discussed. Particular attention has been paid to the analysis of the mutual diffusion coefficient dependence on concentration and temperature. The influence of binary parameters determined either from VLE data or from diffusion experiments, on predicted results is also discussed.

\subsection{Calculation of thermodynamic quantities}

In this study, two $g^{E}$ models, NRTL and Wilson models, have been used to obtain the relevant thermodynamic properties related to diffusion, i.e. excess Gibbs energy, and thermodynamic factor. The details of the calculation of the binary interaction parameters of the NRTL and Wilson models, and the relevant thermodynamic quantities for the studied mixture are given in the supplementary material. Here we only shortly present the results of calculations and the analysis of the behavior of these variables for each of the $g^{\mathrm{E}}$ models used.

In order to calculate the relevant thermodynamic properties, binary interaction parameters should be evaluated at the experimental temperature. In this study, we have measured mutual diffusion coefficients for the mixture of interest at 4 temperatures, $298.15 \mathrm{~K}, 303.15 \mathrm{~K}, 308.15 \mathrm{~K}$, and $313.15 \mathrm{~K}$.

Figure 5 shows (a) the excess Gibbs energy, and (b) thermodynamic factor, calculated using the NRTL model. The general impression is that the concentration dependence is much stronger than the temperature dependence. In Figure 5(a), it is shown that $\mathrm{g}^{\mathrm{E}}$ vanishes in the infinite dilution limits, as every other excess property. According to this model, the minimum dimensionless excess Gibbs energy is $\approx-0.50$ and it is in the range 0.4-0.5 IL mole fraction for this mixture. From both sides of this point, it has an increasing behavior until it vanishes at the limit of the concentration range. Figure 5 (b) shows the calculated thermodynamic factor using this model. As expected, $\Gamma$ goes to the unity in the infinite dilution limits. It has an increasing behavior for small IL content, it reaches a maximum at approximately $0.3 \mathrm{IL}$ mole fraction and then gradually decreases. It is important to note the difference in the behavior in comparison with the excess Gibbs energy. While the latter has a decreasing behavior for low concentrations, the opposite occurs with the thermodynamic factor. 

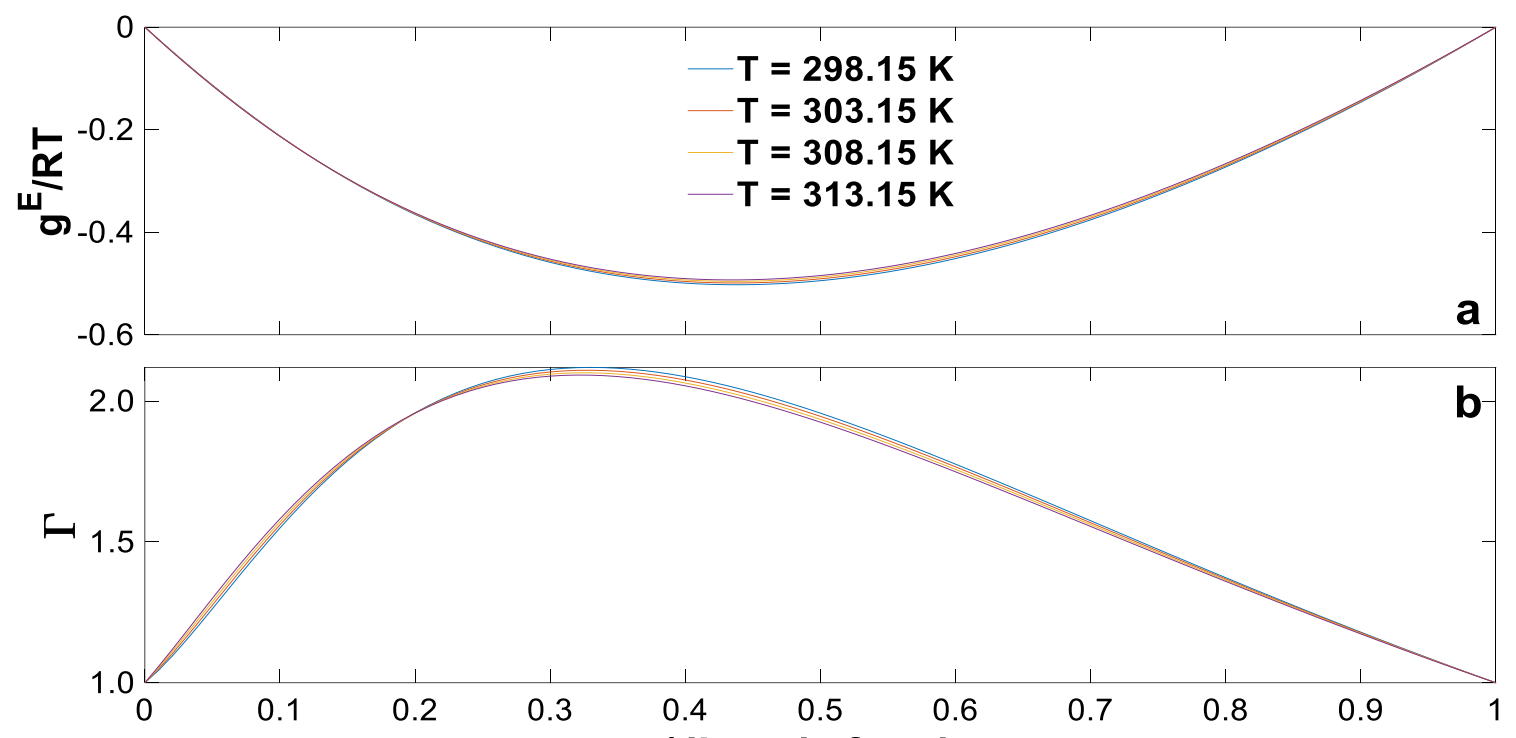

$\mathbf{x} /$ IL mole fraction

Figure 5. (a)Excess Gibbs energy, and (b)thermodynamic factor calculated using the NRTL model with parameters fitted to VLE data for the mixture $\mathrm{H}_{2} \mathrm{O}+\left[\mathrm{EtOH}\right.$ mim][BF $\left.\mathrm{F}_{4}\right]$

4 For the Wilson model, as it was in the case of the NRTL model, the binary interaction parameters were evaluated at different experimental temperatures at which diffusion coefficients were measured. Figure 6(a) shows the calculated excess Gibbs energy using the Wilson model. According to this model, the minimum dimensionless excess Gibbs energy for this mixture is $\approx-0.56$ and it occurs around $0.5 \mathrm{IL}$ mole fraction. Figure $6(\mathrm{~b})$ shows the calculated thermodynamic factor using this model. The behavior of the thermodynamic factor is similar to that of the NRTL model, although is worth noting that its value is smaller, and the temperature dependence is more pronounced. The maximum value of the thermodynamic factor is slightly displaced towards higher concentrations and is around $0.6 \mathrm{IL}$ mole fraction.
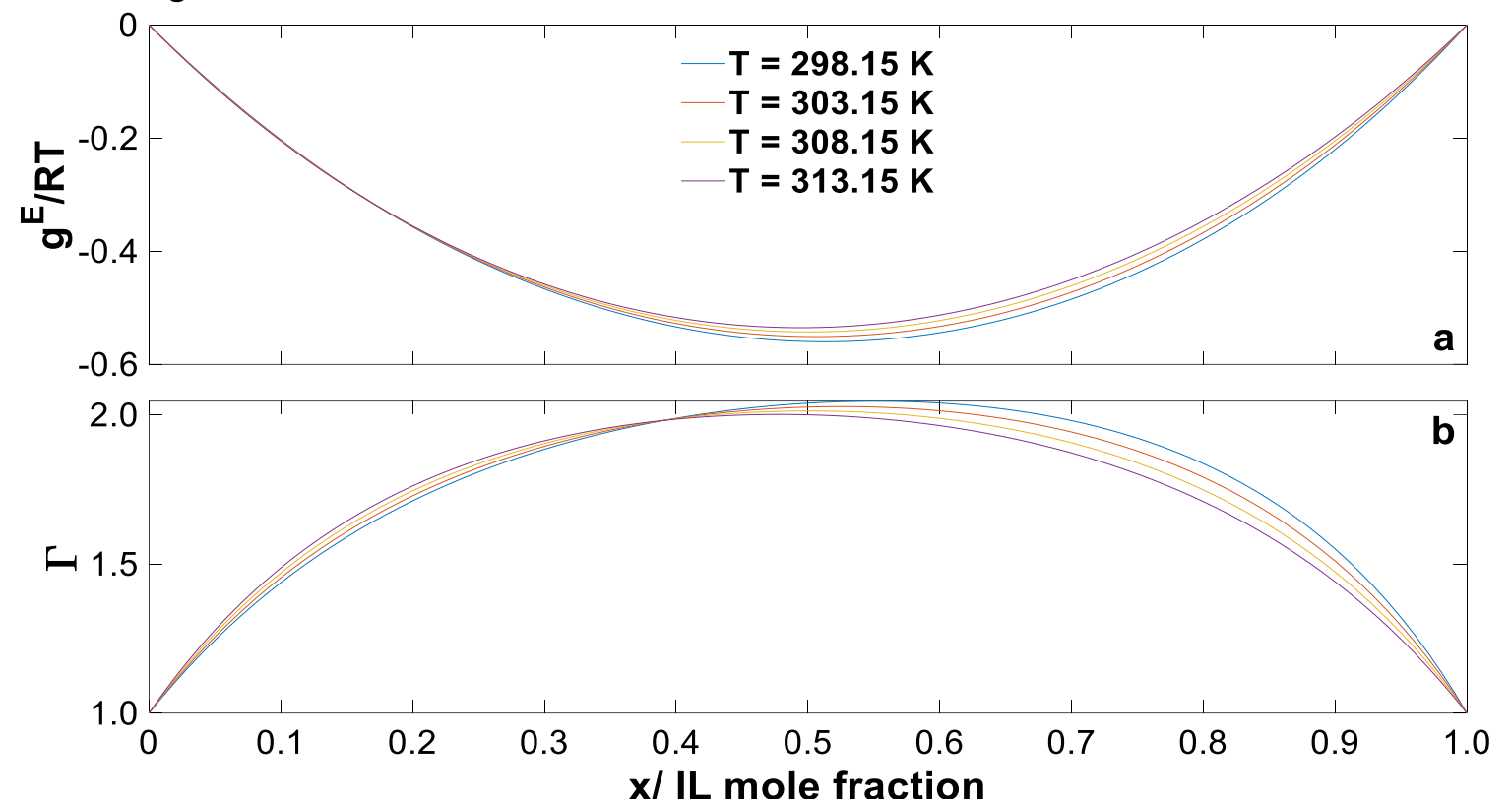

Figure 6. (a)Excess Gibbs energy, and (b)thermodynamic factor calculated using the Wilson model with parameters fitted to VLE data for the mixture $\mathrm{H}_{2} \mathrm{O}+[\mathrm{EtOHmim}]\left[\mathrm{BF}_{4}\right]$. 


\subsection{Experimental diffusion results}

In this work, the mutual diffusion coefficient of a binary mixture of ionic liquid $\mathrm{H}_{2} \mathrm{O}+$ [EtOHmim] [BF4] at temperature 298.15-313.15 $\mathrm{K}$ was measured using optical digital interferometry. As detailed in Section 3.3, two different routes were used to determine the mutual diffusion coefficient from experimentally sampled data. As an example, fitting results are discussed for the experimental measurement at $T=308.15 \mathrm{~K}$ and $\omega$ $=0.500 \mathrm{~kg} \mathrm{~kg}^{-1}$.

Figure 7(a) shows refractive index profiles at two time instances, one of which (the black curve) corresponds to the profile at an early stage of diffusion, and the other one corresponds to the upper limit of the short-time model. The diffusion front reached the top and bottom of the diffusion cell at the observation time $t=3$ hours, which means that the experimental data at later times can only be used for the longtime model. Note that the diffusion experiments lasted for about 15 hours at each state point. Figure $7(\mathrm{~b})$ illustrates the fitting result of the long-time model. The successive curves show the time evolution of the refractive index change along the cell height. The fitting results obtained by the short-time and long-time models are very similar, i.e. $D=[7.53$ and 7.57$] \times 10^{-10} \mathrm{~m}^{2} \mathrm{~s}^{-1}$, respectively

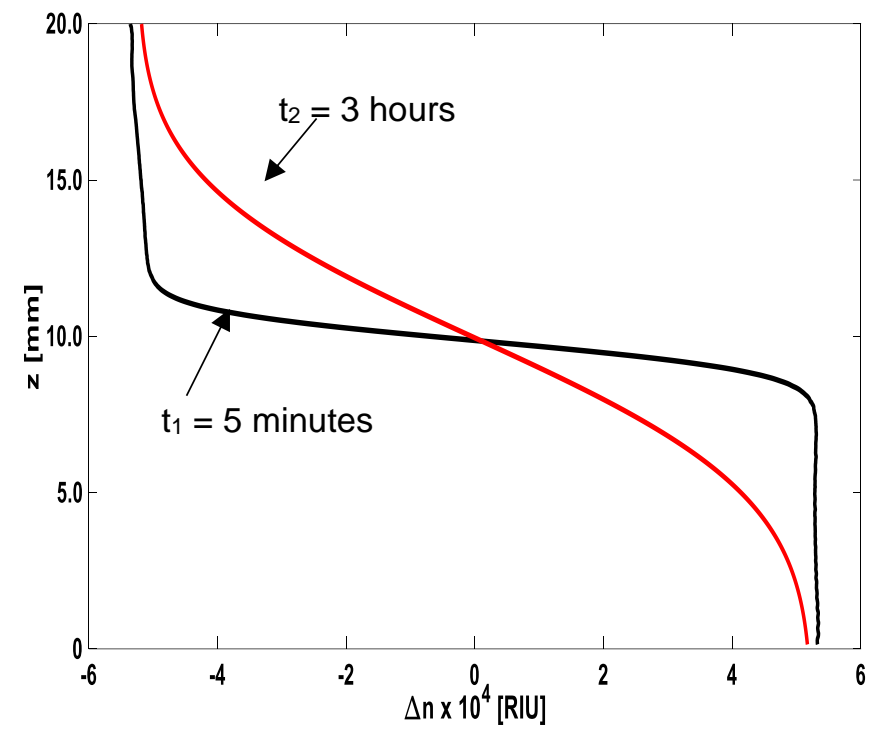

a)

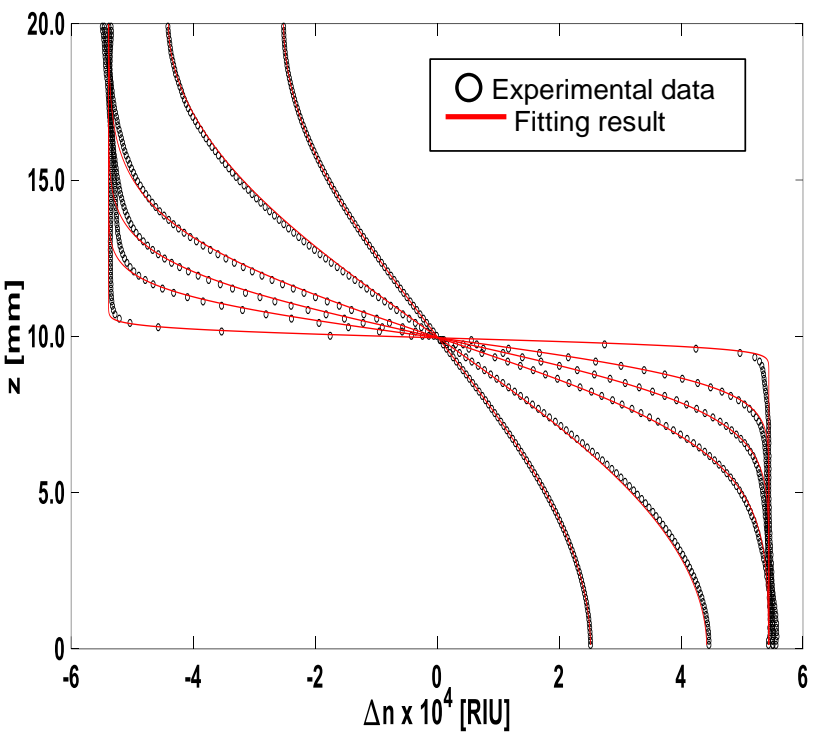

b)

Figure 7. Variation of the refractive index $(\Delta n(z, t))$ along the cell in different time instances for the mixture $\mathrm{H}_{2} \mathrm{O}+\left[\mathrm{EtOHmim}_{[}\left[\mathrm{BF}_{4}\right]\right.$. a) Experimental data on short-time scale; $b$ ) Experimental data and fitting by long-time model.

Table 2 presents the experimental results of the mutual diffusion coefficient and its uncertainties at different IL mass fractions and temperatures.

Table 2. Measured mutual diffusion coefficients $\left(D_{12} \times 10^{10}\left[\mathrm{~m}^{2} \mathrm{~s}^{-1}\right]\right)$ of $\mathrm{H}_{2} \mathrm{O}+\left[\right.$ EtOHmim] $\left[\mathrm{BF}_{4}\right]$ mixtures at different IL mass fraction, $\omega$, and temperature, $T$.

\begin{tabular}{|c|c|c|c|c|}
\hline $\mathbf{\omega}$ & $\mathbf{T}=\mathbf{2 9 8 . 1 5} \mathbf{K}$ & $\mathbf{T}=\mathbf{3 0 3 . 1 5} \mathbf{K}$ & $\mathbf{T}=\mathbf{3 0 8 . 1 5} \mathbf{K}$ & $\mathbf{T}=\mathbf{3 1 3 . 1 5} \mathbf{K}$ \\
\hline 0.0060 & $10.19 \pm 0.05$ & $11.03 \pm 0.05$ & $11.87 \pm 0.05$ & $12.72 \pm 0.05$ \\
\hline 0.1000 & $8.93 \pm 0.05$ & & & \\
\hline 0.2000 & $7.84 \pm 0.04$ & $8.74 \pm 0.05$ & $9.74 \pm 0.04$ & $10.47 \pm 0.04$ \\
\hline 0.3000 & $6.92 \pm 0.04$ & & & \\
\hline
\end{tabular}




\begin{tabular}{|l|l|l|l|l|}
\hline 0.4000 & $5.87 \pm 0.05$ & & & \\
\hline 0.5000 & $5.80 \pm 0.03$ & $6.37 \pm 0.03$ & $7.57 \pm 0.04$ & $8.80 \pm 0.03$ \\
\hline 0.6000 & $5.74 \pm 0.03$ & & & \\
\hline 0.7000 & $5.70 \pm 0.03$ & & & \\
\hline 0.8000 & $5.34 \pm 0.05$ & $6.12 \pm 0.05$ & $7.11 \pm 0.05$ & $7.83 \pm 0.06$ \\
\hline 0.9000 & $4.05 \pm 0.10$ & & & \\
\hline 0.9975 & $2.04 \pm 0.10$ & $2.21 \pm 0.11$ & $2.42 \pm 0.11$ & $3.01 \pm 0.13$ \\
\hline
\end{tabular}

\subsubsection{Concentration and temperature dependences of the mutual diffusion coefficient}

One of the targets of our research was aimed at the examination of the concentration dependence of the mutual diffusion coefficient. The literature data reported [25] that in some cases the mutual diffusion coefficient varies linearly between the two limiting diffusion coefficients, while in others strong positive or negative deviations from linearity are observed.

Figure 8 shows the measured mutual diffusion coefficient of $\mathrm{H}_{2} \mathrm{O}+[\mathrm{EtOHmim}]\left[\mathrm{BF}_{4}\right]$ as a function of IL mass fraction. We found that with the increase of IL mass fraction the mutual diffusion coefficient diminishes. It can be related to the opposite effect of the viscosity on the diffusion process in binary solutions. The viscosity of this IL is considerably higher than that of $\mathrm{H}_{2} \mathrm{O}$, therefore, by increasing the $\mathrm{IL}$ mass fraction the solution viscosity increases rapidly. Over the concentration range the mutual diffusion coefficient displays different types of behavior. For IL content in the mixture below 0.40 , the mutual diffusion coefficient decreases almost linearly with mass fraction. For the composition range $0.40<\omega<0.80$, mutual diffusion decreases very slowly. This behavior is relevant for absorption systems, which generally work in this composition range (0.50-0.80 of absorbent mass fraction). At a higher content of [EtOHmim][BF4] $(\omega>0.80)$, the mutual diffusion coefficient decreases sharply because the viscosity of the solution increases abruptly. It is worth emphasizing that the viscosity of the pure ionic liquid is several tens of times greater than that of water in the studied temperature range.

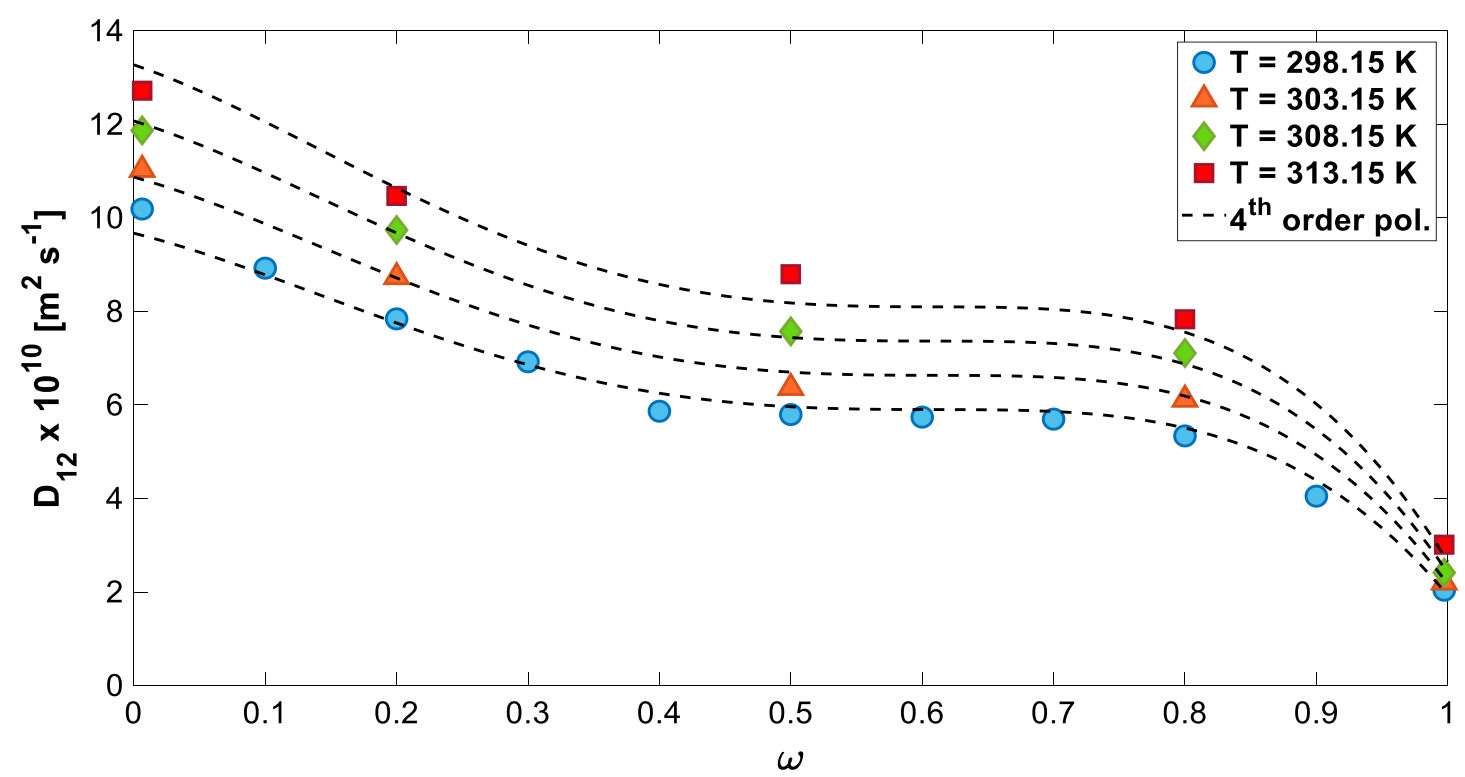

Figure 8. Measured mutual diffusion coefficient at different temperatures as a function of composition; $\omega$ is the mass fraction of ionic liquid. 
Composition dependence of the measured mutual diffusion can be very well correlated with fourth-order polynomials, which are represented by dashed lines in Figure 8. The similarity in the behavior of the curves at the four experimental temperatures is clear. According to our results, the concentration and temperature dependence of the mutual diffusion coefficient is described as:

$$
D_{12}(\omega, \mathrm{T}) \times 10^{10}=D_{0}\left[1+\alpha\left(\mathrm{T}-T_{0}\right)\right]
$$

where:

$$
D_{0}(\omega) \times 10^{10}=a \omega_{1}^{4}+b \omega_{1}^{3}+c \omega_{1}^{2}+d \omega_{1}+D_{1}^{\infty}
$$

Here, $a, b, c, d$ are the polynomial coefficients; $D_{1}^{\infty}$ represents the diffusion coefficient of [EtOHmim] [BF4] at infinite dilution in $\mathrm{H}_{2} \mathrm{O}$ at reference temperature $T_{0}$ (in this work, $T_{0}=298.15 \mathrm{~K}$ ); and $\alpha$ is an empirical parameter, which gives a sense of the percentage of the diffusion coefficient variation per temperature variation of $1 \mathrm{~K}$. Coefficients of Eqs. $D 12 \omega, T \times 10^{10}=D_{0}\left[1+\alpha\left(T-T_{0}\right)\right] \quad$ (21) and $\quad\left(D \square 0 \square(\omega) \times 10^{10}=a \omega_{1}^{4}+\right.$ $b \omega_{1}^{3}+c \omega_{1}^{2}+d \omega_{1}+D_{1}^{\infty} \quad$ (22) were obtained by least square regression from measured diffusion coefficients. These coefficients and quality of fit $\left(R^{2}\right)$ are listed in Table 3.

Table 3. Coefficients of Eqs. (D国2回 $(\omega, \mathrm{T}) \times 10^{10}=D_{0}\left[1+\alpha\left(\mathrm{T}-T_{0}\right)\right]$

\begin{tabular}{|l|c|c|}
\hline \multicolumn{1}{|c|}{$a \omega_{1}^{4}+b \omega_{1}^{3}+c \omega_{1}^{2}+d \omega_{1}+D_{1}^{\infty}$} \\
\hline Coefficient & Value \\
\hline$a$ & -50.42 \\
\hline$b$ & 75.85 \\
\hline$c$ & -26.18 \\
\hline$d$ & -6.993 \\
\hline$D_{1}^{\infty}$ & 9.673 \\
\hline$\alpha$ & 0.02482 \\
\hline$R^{2}$ & 0.9918 \\
\hline
\end{tabular}

(21) and (D曰0 0 ( $(\omega) \times 10^{10}=$

The absolute relative deviation (ARD) was used as the main criterion to evaluate the prediction ability of $D 0 \omega \times 10^{10}=a \omega_{1}^{4}+b \omega_{1}^{3}+c \omega_{1}^{2}+d \omega_{1}+D_{1}^{\infty} \quad$ (22).

$$
A R D \%=\frac{100}{N} \sum_{i=1}^{N}\left|\frac{D_{\exp }-D_{\text {cal }}}{D_{\text {exp }}}\right|
$$

where $N$ is the number of experimental diffusion points.

At the four studied temperatures, the ARD was found to be always less than $4.2 \%$.

We have also investigated the effect of the temperature on the mutual diffusion coefficient of $\mathrm{H}_{2} \mathrm{O}+\left[\mathrm{EtOHmim}_{[}\left[\mathrm{BF}_{4}\right]\right.$ at selected compositions $\omega=(0.006 ; 0.20 ; 0.50$; $0.80 ; 0.9975)$. As expected, with the increase of the temperature the mutual diffusion coefficient grows up due to intensified molecular thermal motion. Figure 9 shows the mutual diffusion coefficient of the studied mixture as a function of temperature at the selected compositions. 


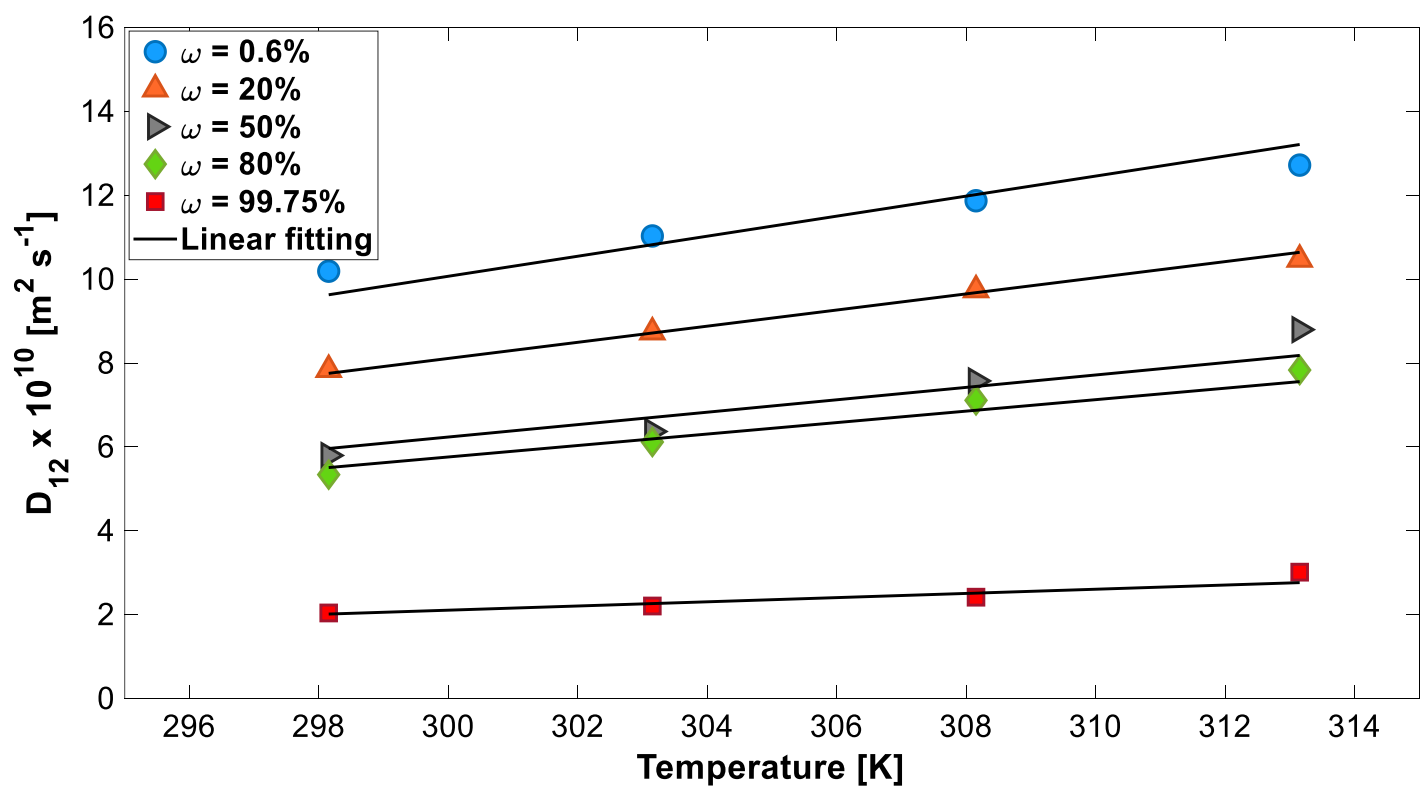

Figure 9. Measured mutual diffusion coefficient at different IL mass fractions $(\omega)$.

In Figure 9, it is possible to appreciate the linear relationship between the mutual diffusion coefficient and temperature in the studied experimental conditions. Wong et al. [35] also reported the linear dependency for [emim, (1-ethyl-3methylimidazolium)]-based ionic liquids in water at infinite dilution for temperatures ranging from 303.2 to $323.2 \mathrm{~K}$ using Taylor dispersion method. Here, the largest but still reasonable deviation was observed in the case $\omega=0.9975(\mathrm{ARD}=4.9 \%)$. Thus, a linear fitting provides correct and important trends for the temperature dependence of the mutual diffusion coefficient.

\subsection{Predictive diffusion results}

In this work, we have measured the mutual diffusion coefficient for the mixture $\mathrm{H}_{2} \mathrm{O}+$ [EtOHmim] $\left[\mathrm{BF}_{4}\right]$ at different IL mass fractions and temperatures. In total, we have 26 experimental points in the full range of concentration. In particular, mutual diffusion coefficients at $T=298.15 \mathrm{~K}$ were measured at intervals of $0.10 \mathrm{IL}$ mass fraction. As soon as we recognized that the concentration dependence of the mutual diffusion coefficient for this mixture can be reliably fitted by fourth-order polynomials, the need for a larger number of experimental points vanished. Thus, we used these polynomials to obtain the mutual diffusion coefficient at intervals of 0.10 of IL mass fraction for the other 3 experimental temperatures, $T=(303.15 ; 308.15$; and 313.15$)$ $\mathrm{K}$. From this point on, the calculated mutual diffusion coefficients were treated as experimental points. Therefore, instead of 26 experimental diffusion points, we extended them to 44 experimental points.

Prior to comparing the performance of different predictive models, we need to determine the optimal binary interaction parameters. First, we determined these binary parameters by means of VLE data regression using $g^{\mathrm{E}}$ models. Next, we used the binary parameters as adjustable parameters with experimental diffusion data. A comparison between the results using both strategies is made, and some conclusions are drawn. 
In this case, the parameters obtained from VLE data (see in the supplementary materials Table1s and Table2s for NRTL and Wilson models, respectively) are used to calculate the thermodynamic factor, $\Gamma$. The binary parameters are evaluated at all experimental temperatures.

We found that the classical approach of Vignes does not provide satisfactory results of the mutual diffusion coefficient for the mixture of $\mathrm{H}_{2} \mathrm{O}+[\mathrm{EtOHmim}]\left[\mathrm{BF}_{4}\right]$. The deviation of the mutual diffusion coefficient, estimated by Vignes correlation when using NRTL and Wilson models to calculate the thermodynamic factor, from our experimental results is $76.8 \%$ and $70.4 \%$, respectively. In general, the estimated values are much higher than the experimental ones. This trend was previously observed for classical binary systems [36]. The difference of $6 \%$ between the values estimated by NRTL or Wilson models for Vignes correlation can be attributed to the fact that the thermodynamic factor is higher with NRTL model than with Wilson model for most experimental points (see figures Figure 5 and Figure 6).

We also examined the diffusion coefficients obtained with UNIDIF model. This model has two adjustable parameters which can be determined from diffusion data measured in this work. Therefore, its results will be presented in the next section.

Now, we will shortly describe the results obtained with the cluster diffusion model, where binary parameters for determination of thermodynamic factor are calculated using NRTL and Wilson models from VLE data. In addition, from section 2.3 follows that 5 adjustable parameters must be determined in order to predict diffusion coefficients. The cluster model requires knowledge of measured diffusion coefficients to regress adjustable parameters. Using 5 parameters fit procedure, we have determined the optimal values of adjustable parameters, which are listed in Table $3 \mathrm{~s}$ in the supplementary material. Using these optimal adjustable parameters and binary data, we have calculated diffusion coefficients $\left(D_{\text {cal }}\right)$ and compared them with the experimental values $\left(D_{\exp }\right)$. The results for $T=298.15 \mathrm{~K}$ are summarized in Table 4 .

Table 4. Comparison of experimental and calculated by cluster diffusion model mutual diffusion coefficients at $T=298.15 \mathrm{~K}$

\begin{tabular}{|c|c|c|c|}
\hline $\begin{array}{c}\omega \\
\text { [IL mass fraction] }\end{array}$ & $\begin{array}{c}\text { Dexp } \mathbf{X} \mathbf{1 0}^{\mathbf{1 0}} \\
{\left[\mathbf{m}^{\mathbf{2}} \mathbf{~ s}^{\mathbf{- 1}} \text { ] }\right.}\end{array}$ & \multicolumn{2}{|c|}{$\left.\mathbf{D}_{\text {cal } \mathbf{~} \mathbf{1 0}} \mathbf{1 0}^{\mathbf{1 0}} \mathbf{m}^{\mathbf{2}} \mathbf{s}^{\mathbf{- 1}}\right]$} \\
\hline 0.0060 & 10.19 & $\mathbf{N R T}$ & Wilson \\
\hline 0.1000 & 8.93 & 9.99 & 8.24 \\
\hline 0.2000 & 7.84 & 8.41 & 8.07 \\
\hline 0.3000 & 6.92 & 7.81 & 7.86 \\
\hline 0.4000 & 5.87 & 7.31 & 7.60 \\
\hline 0.5000 & 5.80 & 6.88 & 6.29 \\
\hline 0.6000 & 5.74 & 6.44 & 6.35 \\
\hline 0.7000 & 5.70 & 5.84 & 5.60 \\
\hline 0.8000 & 5.34 & 4.78 & 4.49 \\
\hline 0.9000 & 4.05 & 2.70 & 2.58 \\
\hline 0.9975 & 2.04 & 0.21 & 0.09 \\
\hline ARD [\%] & - & 18.3 & 18.8 \\
\hline
\end{tabular}


1 The absolute relative deviation (ARD) defined in Eq. (ARD $\%=\frac{100}{N} \sum_{i=1}^{N}\left|\frac{D_{\text {exp }}-D_{\text {cal }}}{D_{\text {exp }}}\right|$ (23) was used as the main criterion to evaluate the prediction ability of the predictive models presented in Section 2.

The cluster diffusion model provides a deviation from experimental data of about $18 \%$. The deviation from experimental data can be explained by the fact that this model was developed for concentrated solutions, unlike the MGC model. In addition, originally this model was developed to calculate binary interaction parameters as adjustable parameters, without considering temperature dependence. It is worth mentioning that viscosity was calculated due to absence of experimental data and the radius of the IL solute was treated as an adjustable parameter. Here, the differences between NRTL (18.3\%) and Wilson (18.8\%) models are negligible. We suggest this is because in the expression developed by Kamgar et al.( $D 12=$ $k 7$ ? $2 \pi \eta(2-n) \beta r_{0}$ 国 $\left(\frac{1}{1+Y^{*} \frac{1}{x_{1} x_{2}}\left(\frac{1}{\Gamma}-1\right)}\right)^{\frac{1}{3-n}}$

(10)), the thermodynamic factor has a smaller contribution to the value of the mutual diffusion coefficient than in Vignes correlation. Note that Kamgar et al. [23] results also displayed a tiny difference between the use of NRTL and Wilson models.

The next considered model, the MGC model, provides much better estimations of the mutual diffusion coefficients. As it was described in section 2.4, the use of MGC model requires 7 adjustable parameters. To determine these adjustable parameters, first we need to calculate the thermodynamic factor, and for this we used NRTL and Wilson models. Except this basic quantity, the MGG model requires knowledge of measured diffusion coefficients to regress adjustable parameters. Finally, having in hands the optimal adjustable parameters, the diffusion coefficients can be calculated in the given range of temperatures and over the full range of composition. We have calculated the best-fit parameters for this model, which are shown in Table $4 \mathrm{~s}$ in the supplementary material. We have calculated diffusion using optimal adjustable parameters, results are given in Table 5. As can be seen, the deviation from experimental data is $7.9 \%$ and $8.8 \%$ when using NRTL and Wilson models, respectively.

Table 5. Comparison of experimental and calculated by MGC model mutual diffusion coefficients at $T=298.15 \mathrm{~K}$.

\begin{tabular}{|c|c|c|c|}
\hline \multirow{2}{*}{$\stackrel{\omega}{[\text { [IL mass fraction] }}$} & \multirow{2}{*}{$\begin{array}{c}D_{\exp } \times 10^{10} \\
{\left[\mathrm{~m}^{2} \mathbf{s}^{-1}\right]}\end{array}$} & \multicolumn{2}{|c|}{$D_{\text {cal }} \times 10^{10}\left[\mathrm{~m}^{2} \mathrm{~s}^{-1}\right]$} \\
\hline & & NRTL & Wilson \\
\hline 0.0060 & 10.19 & 8.93 & 9.05 \\
\hline 0.1000 & 8.93 & 8.47 & 8.54 \\
\hline 0.2000 & 7.84 & 7.96 & 7.99 \\
\hline 0.3000 & 6.92 & 7.44 & 7.42 \\
\hline 0.4000 & 5.87 & 6.91 & 6.85 \\
\hline 0.5000 & 5.80 & 6.39 & 6.28 \\
\hline 0.6000 & 5.74 & 5.89 & 5.71 \\
\hline 0.7000 & 5.70 & 5.46 & 5.17 \\
\hline 0.8000 & 5.34 & 5.20 & 4.71 \\
\hline 0.9000 & 4.05 & 5.30 & 4.57 \\
\hline 0.9975 & 2.04 & 2.25 & 1.01 \\
\hline ARD [\%] & - & 7.9 & 8.8 \\
\hline
\end{tabular}


Among the studied models, with binary parameters regressed from VLE data, the MGC model using the NRTL equations is the one that provides the best results. Figure 10 shows the experimental and predicted mutual diffusion coefficients using this model. For the sake of clarity, only two experimental temperatures are shown, the lowest $(T=298.15 \mathrm{~K})$ and the highest $(T=313.15 \mathrm{~K})$, their behavior is very similar for the other two temperatures. It follows from Figure 10 that the agreement is similar for both temperatures. In regard to the concentration dependence, the tendency is influenced by temperature. For example, at $T=298.15 \mathrm{~K}$, the model overestimates the mutual diffusion coefficient in the range $\omega=0.2-0.5$. At $T=313.15 \mathrm{~K}$, the model underestimates the mutual diffusion coefficient in the range $\omega=0.6-0.8$. The maximum discrepancy between experimental and calculated results does not exceed $8 \%$. Thus, it acceptably reproduces the behavior of the mutual diffusion coefficient in the range of the given experimental parameters.

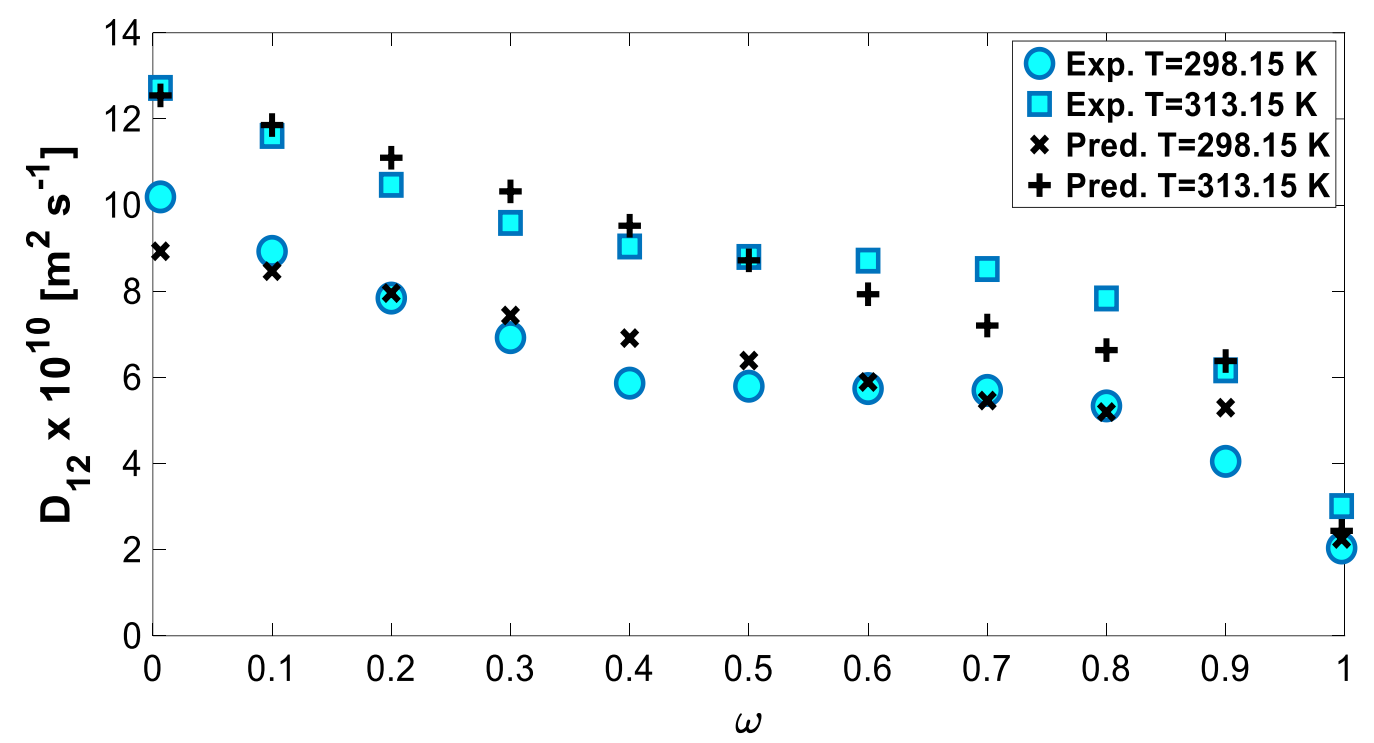

Figure 10. Experimental and predicted mutual diffusion coefficients using MGC model and NRTL equations with fitting to VLE data for mixture $\mathrm{H}_{2} \mathrm{O}+\left[\mathrm{EtOHmim}_{[}\left[\mathrm{BF} \mathrm{F}_{4}\right]\right.$.

\subsubsection{Predictive results with binary parameters regressed from diffusion data}

In this section, the binary parameters used to calculate the thermodynamic factor, $\Gamma$, are regressed from our measured diffusion data.

First, we considered Vignes correlation. The obtained best-fit parameters for this correlation are listed in Table $5 \mathrm{~s}$ in the supplementary material. The diffusion coefficients calculated with such determined parameters are shown in Table 6. It provides a much better estimation than using binary parameters from VLE data. The deviation from experimental data is $17.2 \%$ and $26.5 \%$ when using NRTL and Wilson to calculate the thermodynamic factor, respectively. Still, the classical approach of Vignes does not provide satisfactory estimates for the mutual diffusion coefficient of the studied mixture.

Table 6. Comparison of the mutual diffusion coefficients obtained experimentally and calculated by Vignes correlation at $T=298.15 \mathrm{~K}$
$\omega$
$D_{\exp } \times \mathbf{1 0}^{10}$
$D_{\text {cal }} \times 10^{10}\left[\mathrm{~m}^{2} \mathrm{~s}^{-1}\right]$ 


\begin{tabular}{|c|c|c|c|}
\hline [IL mass fraction] & {$\left[\mathbf{m}^{\mathbf{2}} \mathbf{~ s}^{-1}\right]$} & NRTL & Wilson \\
\hline 0.0060 & 10.19 & 10.16 & 9.61 \\
\hline 0.1000 & 8.93 & 9.77 & 9.36 \\
\hline 0.2000 & 7.84 & 9.33 & 9.04 \\
\hline 0.3000 & 6.92 & 8.87 & 8.65 \\
\hline 0.4000 & 5.87 & 8.36 & 8.18 \\
\hline 0.5000 & 5.80 & 7.78 & 7.58 \\
\hline 0.6000 & 5.74 & 7.06 & 6.80 \\
\hline 0.7000 & 5.70 & 6.11 & 5.76 \\
\hline 0.8000 & 5.34 & 4.79 & 4.32 \\
\hline 0.9000 & 4.05 & 2.96 & 2.29 \\
\hline 0.9975 & 2.04 & 1.97 & 0.04 \\
\hline ARD [\%] & - & 17.2 & 26.5 \\
\hline
\end{tabular}

1

12

The prediction ability of the UNIDIF model has been also tested. As mentioned, the correlation proposed by Hsu and Chen [24] has the particularity that does not involve the use of a thermodynamic factor. Therefore, it is quite different from the other models studied here. It has only two adjustable parameters which are listed in Table $6 s$ in the supplementary material. The diffusion coefficients calculated with these parameters are shown in Table 7 . This model provides a deviation from experimental data of $13.9 \%$.

Table 7. Comparison of the mutual diffusion coefficients obtained experimentally and calculated by UNIDIF model at $T=298.15 \mathrm{~K}$.

\begin{tabular}{|c|c|c|}
\hline $\begin{array}{c}\boldsymbol{\omega} \\
\text { [IL mass fraction] }\end{array}$ & $\begin{array}{c}\mathbf{D}_{\exp \mathbf{~} \mathbf{~ 1 0}} \\
{\left[\mathbf{m}^{\mathbf{2}} \mathbf{~}^{-1} \mathbf{]}\right.}\end{array}$ & $\begin{array}{c}\mathbf{D}_{\text {cal }} \mathbf{X} \mathbf{~ 1 0}^{\mathbf{1 0}} \\
{\left[\mathbf{m}^{\mathbf{2}} \mathbf{~ s}^{-1}\right]}\end{array}$ \\
\hline 0.0060 & 10.19 & 10.16 \\
\hline 0.1000 & 8.93 & 9.70 \\
\hline 0.2000 & 7.84 & 9.16 \\
\hline 0.3000 & 6.92 & 8.55 \\
\hline 0.4000 & 5.87 & 7.88 \\
\hline 0.5000 & 5.80 & 7.11 \\
\hline 0.6000 & 5.74 & 6.25 \\
\hline 0.7000 & 5.70 & 5.27 \\
\hline 0.8000 & 5.34 & 4.17 \\
\hline 0.9000 & 4.05 & 3.01 \\
\hline 0.9975 & 2.04 & 2.05 \\
\hline ARD [\%] & - & 13.9 \\
\hline
\end{tabular}

We also verified the prediction ability of the cluster diffusion model with binary parameters from diffusion data. All the adjustable parameters for this model are listed in Table $7 \mathrm{~s}$ in the supplementary material. Diffusion coefficients calculated with this model provide very good results as shown in Table 8 . In this case, the deviation from experimental data is $4.1 \%$ and $3.4 \%$ for NRTL and Wilson models, respectively.

Table 8. Comparison of the mutual diffusion coefficients obtained experimentally and calculated by cluster model at $T=298.15 \mathrm{~K}$ (using binary parameters from diffusion data)

\begin{tabular}{|c|c|c|c|}
\hline $\begin{array}{c}\omega \\
\text { [IL mass fraction] }\end{array}$ & $\begin{array}{c}\mathbf{D}_{\text {exp }} \mathbf{X} \mathbf{1 0}^{\mathbf{1 0}} \\
{\left[\mathbf{m}^{\mathbf{2}} \mathbf{~ s}^{-1}\right]}\end{array}$ & \multicolumn{2}{|c|}{$\mathbf{D}_{\text {cal }} \mathbf{X} \mathbf{1 0}^{\mathbf{1 0}}\left[\mathbf{m}^{\mathbf{2}} \mathbf{~ s}^{-1}\right]$} \\
\cline { 3 - 4 } & 10.190 & NRTL & Wilson \\
\hline 0.0060 & 8.925 & 10.61 & 9.95 \\
\hline 0.1000 & 7.841 & 7.43 & 8.86 \\
\hline 0.2000 & & & 7.83 \\
\hline
\end{tabular}




\begin{tabular}{|c|c|c|c|}
\hline 0.3000 & 6.923 & 6.69 & 6.98 \\
\hline 0.4000 & 5.866 & 6.17 & 6.34 \\
\hline 0.5000 & 5.795 & 5.82 & 5.94 \\
\hline 0.6000 & 5.740 & 5.55 & 5.75 \\
\hline 0.7000 & 5.697 & 5.28 & 5.61 \\
\hline 0.8000 & 5.338 & 4.82 & 5.14 \\
\hline 0.9000 & 4.050 & 3.75 & 3.70 \\
\hline 0.9975 & 2.039 & 1.40 & 1.48 \\
\hline ARD [\%] & - & 4.1 & 3.4 \\
\hline
\end{tabular}

As for the MGC model, we also calculated diffusion coefficients using adjustable parameters from Table $8 \mathrm{~s}$ in the supplementary material. The calculated diffusion coefficients using binary parameters from diffusion data are shown in Table 9. The absolute relative deviation with respect to the experimental data is $2.1 \%$ and $3.4 \%$ when using the NRTL and Wilson equations, respectively. It provides not only very good predictive results, but the results are also superior to those obtained with parameters regressed from VLE data.

Table 9. Comparison of the mutual diffusion coefficients obtained experimentally and calculated by MGC model at $T=298.15 \mathrm{~K}$ (using binary parameters from diffusion data)

\begin{tabular}{|c|c|c|c|}
\hline \multirow{2}{*}{$\begin{array}{c}\omega \\
\text { [IL mass fraction] }\end{array}$} & \multirow{2}{*}{$\begin{array}{c}D_{\exp } \times 10^{10} \\
{\left[\mathrm{~m}^{2} \mathrm{~s}^{-1}\right]}\end{array}$} & \multicolumn{2}{|c|}{$D_{\text {cal }} \times 10^{10}\left[\mathrm{~m}^{2} \mathrm{~s}^{-1}\right]$} \\
\hline & & NRTL & Wilson \\
\hline 0.0060 & 10.190 & 10.40 & 9.80 \\
\hline 0.1000 & 8.925 & 8.85 & 8.88 \\
\hline 0.2000 & 7.841 & 7.65 & 7.97 \\
\hline 0.3000 & 6.923 & 6.79 & 7.14 \\
\hline 0.4000 & 5.866 & 6.18 & 6.41 \\
\hline 0.5000 & 5.795 & 5.78 & 5.81 \\
\hline 0.6000 & 5.740 & 5.57 & 5.41 \\
\hline 0.7000 & 5.697 & 5.50 & 5.28 \\
\hline 0.8000 & 5.338 & 5.41 & 5.46 \\
\hline 0.9000 & 4.050 & 4.27 & 4.56 \\
\hline 0.9975 & 2.039 & 1.88 & 2.05 \\
\hline ARD [\%] & - & 2.1 & 3.4 \\
\hline
\end{tabular}

\subsubsection{Comparison of the performance of the predictive models}

In this study, we examined four predictive models and binary parameters for each of them were estimated using two different methodologies. The results of analysis are summarized in Table 10. The numbers in brackets represent the number of adjustable parameters that are used in each model. It follows from the data in this table that the use of binary interaction parameters from diffusion data considerably improves the prediction ability of all the models, even in the simplest case of Vignes correlation. In line with literature data, our study confirms that among the analyzed models, the MGC model using the NRTL equations, provides the best predictions.

Table 10. Comparison of the error (ARD\%) of the four predictive models

\begin{tabular}{|l|c|c|c|c|}
\hline Model & \multicolumn{2}{|c|}{ From VLE data } & \multicolumn{2}{|c|}{$\begin{array}{c}\text { From diffusion } \\
\text { experiments }\end{array}$} \\
\hline & NRTL & Wilson & NRTL & Wilson \\
\hline Vignes & $76.8(0)$ & $70.4(0)$ & $17.2(5)$ & $26.5(4)$ \\
\hline Cluster & $18.3(5)$ & $18.7(5)$ & $4.1(10)$ & $3.4(9)$ \\
\hline
\end{tabular}




\begin{tabular}{|c|c|c|c|c|}
\hline MGC & $7.9(7)$ & $8.8(7)$ & $2.1(12)$ & $3.4(11)$ \\
\hline UNIDIF & \multicolumn{2}{|c|}{$13.9(2)$} & \\
\hline
\end{tabular}

In order to highlight the performance of the MGC model, we present in Figure 11 our experimental data and those calculated by this model using NRTL equations. Obviously, this model can not only provide estimates of the mutual diffusion coefficient for the mixture $\mathrm{H}_{2} \mathrm{O}+[\mathrm{EtOHmim}]\left[\mathrm{BF}_{4}\right]$ with great precision but also is capable of reproducing the behavior of the mutual diffusion coefficient as a function of the concentration.

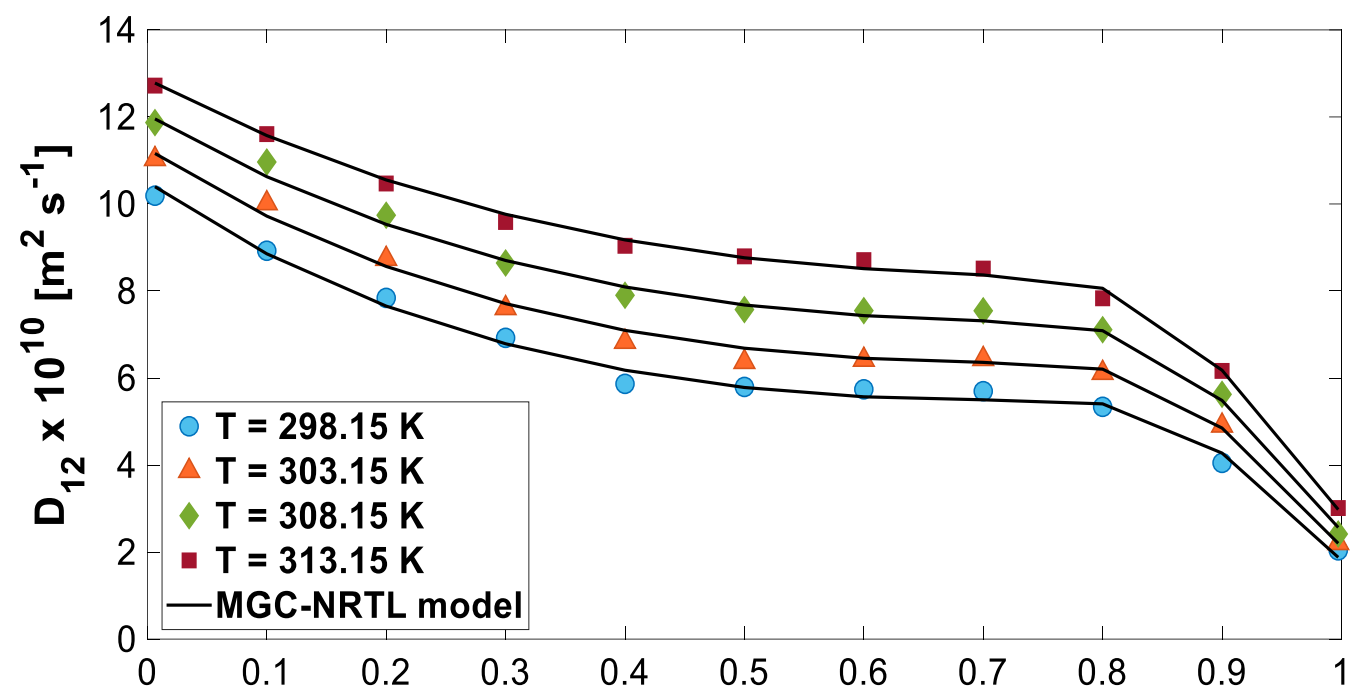

Figure 11. Mutual diffusion coefficients for mixture $\mathrm{H}_{2} \mathrm{O}+[$ [EtOHmim][BF 4 . Symbols: experimental data. Lines: calculations by the MGC model and NRTL equations with binary parameters from diffusion data.

Finally, we have used the regressed binary parameters from diffusion data to calculate the excess Gibbs energy, and the thermodynamic factor. Again, the behavior of the excess Gibbs energy and the thermodynamic factor are opposed as in the case when using binary parameters from VLE data. We have found that the predictive model with the best estimates of the mutual diffusion coefficient, i.e. MGC with NRTL equations, corresponds to the minimum excess Gibbs energy, and thus the maximum thermodynamic factor. Perhaps, it is a general trend but, on this step, we cannot prove it as an examination of different liquid mixtures is required.

\section{Conclusions}

This work is aimed at understanding the behavior of the mutual diffusion coefficient of ionic liquid binary mixtures of $\mathrm{H}_{2} \mathrm{O}+[\mathrm{EtOHmim}]\left[\mathrm{BF}_{4}\right]$. Advanced optical technique, in particular optical digital interferometry, was used to detect the changes in the refractive index in a liquid mixture in order to describe correctly the concentration fields. The mutual diffusion coefficient of a binary mixture of $\mathrm{H}_{2} \mathrm{O}+[\mathrm{EtOHmim}]\left[\mathrm{BF}_{4}\right]$ was measured in the whole composition range and in the temperature range $T=$ 298.15-313.15 K. In addition, four predictive models were employed to describe the mutual diffusion coefficients of this mixture: Vignes, UNIDIF, Modified Group Contribution, and Cluster diffusion. 
As the general trend, the mutual diffusion coefficient of $\mathrm{H}_{2} \mathrm{O}+\left[\mathrm{EtOHmim}^{-}[\mathrm{BF} 4]\right.$ decreases with increasing the ionic liquid mass fraction. It was found that the rate of the decrease is essential only in regions that are IL-poor or IL-rich. In the mid-range, $0.4<\omega<0.8$, the mutual diffusion coefficient is nearly constant. It was also shown that the measured diffusion coefficients can be perfectly described using fourth-order polynomials as a function of the IL mass fraction.

We have also studied the dependence of the mutual diffusion coefficient of $\mathrm{H}_{2} \mathrm{O}+$ $[\mathrm{EtOHmim}]\left[\mathrm{BF}_{4}\right]$ on temperature. The results showed an almost linear increase in the diffusion coefficient with temperature. Linearity is slightly deviated with increasing the mass fraction of the ionic liquid.

A qualitative and quantitative comparison between the experiments and predictions with four different models was performed. Before comparing the performance of

Using binary parameters from VLE data regression (the first method), none of the four models was able to reproduce successfully the dependence of the mutual diffusion coefficient on the IL mass fraction. The use of binary parameters from experimental diffusion data (the second method) improves considerably the prediction ability of the examined predictive models. However, it requires knowledge of the experimental data in wide composition range in advance. We also noticed that for each predictive model employed here, the use of NRTL or Wilson does not lead to significant difference in the results; the mutual diffusion coefficients have the same order of accuracy. However, the difference in predicted diffusion coefficients between the models is essential.

Among the four examined predictive models, only the MGC model provides satisfactory estimates of the mutual diffusion coefficient: an overall ARD from experimental data of $7.9 \%$ using binary interaction parameters regressed from VLE data and $2.1 \%$ using binary parameters regressed from diffusion data.

The Vignes model provides the poorest prediction, its ARD is above $70 \%$ using VLE data regression. However, the Vignes model has a distinct advantage; it requires prior knowledge of experimental diffusion coefficients only in dilute limits, i.e., at two state points and can be used as an initial guess for other models. 
It is expected that the other predictive correlations presented in this paper and, which themselves require experimental diffusion data, can be also employed to predict the coefficients at temperatures which are experimentally more challenging.

[1] X. Zhang, D. Hu, Performance simulation of the absorption chiller using water and ionic liquid 1-ethyl3-methylimidazolium dimethylphosphate as the working pair, Appl. Therm. Eng. 31 (2011) 3316-3321. doi:10.1016/j.applthermaleng.2011.06.011.

[2] M. Shokouhi, M. Adibi, A.H. Jalili, M. Hosseini-jenab, A. Mehdizadeh, Solubility and Diffusion of H2S and $\mathrm{CO} 2$ in the Ionic Liquid 1-(2-Hydroxyethyl)-3-methylimidazolium Tetrafluoroborate, J. Chem. Eng. Data. 55 (2010) 1663-1668.

[3] S. Seki, S. Tsuzuki, K. Hayamizu, Y. Umebayashi, N. Serizawa, K. Takei, H. Miyashiro, Comprehensive refractive index property for room-temperature ionic liquids, J. Chem. Eng. Data. 57 (2012) 2211-2216. doi:10.1021/je201289w.

[4] M. Seiler, A. Kühn, F. Ziegler, X. Wang, Sustainable cooling strategies using new chemical system solutions, Ind. Eng. Chem. Res. 52 (2013) 16519-16546. doi:10.1021/ie401297u.

[5] M. Watanabe, M.L. Thomas, S. Zhang, K. Ueno, T. Yasuda, K. Dokko, Application of Ionic Liquids to Energy Storage and Conversion Materials and Devices, Chem. Rev. 117 (2017) 7190-7239. doi:10.1021/acs.chemrev.6b00504.

[6] Y. Arosa, C.D. Rodríguez Fernández, E. López Lago, A. Amigo, L.M. Varela, O. Cabeza, R. de la Fuente, Refractive index measurement of imidazolium based ionic liquids in the Vis-NIR, Opt. Mater. (Amst). 73 (2017) 647-657. doi:10.1016/j.optmat.2017.09.028.

[7] X. Liu, P. Pan, M. He, Vapor-liquid equilibrium and diffusion coefficients of R32 + [HMIM][FEP], R152a + [HMIM][FEP] and R161 + [HMIM][FEP], J. Mol. Liq. 253 (2018) 28-35. doi:10.1016/j.molliq.2018.01.032.

[8] A. Cera-Manjarres, D. Salavera, A. Coronas, Vapour pressure measurements of ammonia/ionic liquids mixtures as suitable alternative working fluids for absorption refrigeration technology, Fluid Phase Equilib. 476 (2018) 48-60. doi:10.1016/j.fluid.2018.01.006.

[9] H.M. Ariyadi, A. Coronas, Absorption Capacity of Ammonia into Ionic Liquids for Absorption Refrigeration Applications, J. Phys. Conf. Ser. 745 (2016) 032105. doi:10.1088/17426596/745/3/032105.

[10] H.M. Ariyadi, A. Cera-Manjarres, A. Coronas, Absorption Behaviour and Diffusivity of Ammonia in Ionic Liquids, in: 5th IIR Int. Conf. Thermophys. Prop. Transf. Process. Refrig., International Institute of Refrigeration, Seoul, South Korea, 2017: pp. 1-8. doi:10.18462/iir.tptpr.2017.0129.

[11] A. Yokozeki, M.B. Shiflett, Absorption Cycles using H2O + Ionic Liquid Systems, Ind. Eng. Chem. Res. 49 (2010) 9496-9503.

[12] N. Nie, D. Zheng, L. Dong, Y. Li, Thermodynamic Properties of the Water + 1-(2-Hydroxylethyl)-3methylimidazolium Chloride System.pdf, J. Chem. Eng. Data. 57 (2012) 3598-3603.

[13] L. Jing, Z. Danxing, F. Lihua, W. Xianghong, D. Li, Vapor Pressure Measurement of the Ternary Systems $\mathrm{H} 2 \mathrm{O}+\mathrm{LiBr}+[$ Dmim $] \mathrm{Cl}, \mathrm{H} 2 \mathrm{O}+\mathrm{LiBr}+[$ Dmim $] \mathrm{BF} 4, \mathrm{H} 2 \mathrm{O}+\mathrm{LiCl}+[\mathrm{Dmim}] \mathrm{Cl}$, and $\mathrm{H} 2 \mathrm{O}+$ $\mathrm{LiCl}+[$ Dmim]BF4, J. Chem. Eng. Data. 56 (2011) 97-101.

[14] 2013-Huang_Vapor-Liquid Equilibrium Measurements of NH3 + H2O + Ionic Liquid ([Dmim]Cl, [Dmim]BF4, and [Dmim]DMP) Systems.pdf, n.d.

[15] W. Huang, D. Zheng, C. Xia, L. Feng, L. Dong, P. Jiang, Affinity regulation of the NH3 + H2O system by ionic liquids with molecular interaction analysis, Phys. Chem. Chem. Phys. 19 (2017) 16242-16250. 
doi:10.1039/c7cp01757j.

[16] A. Yokozeki, M.B. Shiflett, Water solubility in ionic liquids and application to absorption cycles, Ind. Eng. Chem. Res. 49 (2010) 9496-9503. doi:10.1021/ie1011432.

[17] V. Sechenyh, J.C. Legros, A. Mialdun, J.M. Ortiz De Zárate, V. Shevtsova, Fickian Diffusion in Ternary Mixtures Composed by 1,2,3,4-Tetrahydronaphthalene, Isobutylbenzene, and n-Dodecane, J. Phys. Chem. B. 120 (2016) 535-548. doi:10.1021/acs.jpcb.5b11143.

[18] A. Mialdun, V. Yasnou, V. Shevtsova, Measurement of isothermal diffusion coefficients in ternary mixtures using counter flow diffusion cell, Comptes Rendus - Mec. 341 (2013) 462-468. doi:10.1016/j.crme.2013.02.001.

[19] A. Mialdun, V. Shevtsova, Measurement of the Soret and diffusion coefficients for benchmark binary mixtures by means of digital interferometry, J. Chem. Phys. 134 (2011) 1-12. doi:10.1063/1.3546036.

[20] T. Janzen, S. Zhang, A. Mialdun, G. Guevara-Carrion, J. Vrabec, M. He, V. Shevtsova, Mutual diffusion governed by kinetics and thermodynamics in the partially miscible mixture methanol + cyclohexane, Phys. Chem. Chem. Phys. 19 (2017) 31856-31873. doi:10.1039/c7cp06515a.

[21] A. Vignes, Diffusion in Binary Solutions. Variation of Diffusion Coeffcient with Composition., Ind. Eng. Chem. Fundam. 5 (1966) 189-199.

[22] W. Chen, B. Zhang, Z. Liu, M. Zhao, Z. Miao, Investigation of the mutual diffusion coefficients of [mmim] DMP/H2O and [mmim]DMP/CH3OH at atmospheric pressure, Int. J. Heat Mass Transf. 111 (2017) 559-569. doi:10.1016/j.ijheatmasstransfer.2017.04.025.

[23] A. Kamgar, N. Hamedi, S. Mohsenpour, M.R. Rahimpour, Investigation of using different thermodynamic models on prediction ability of mutual diffusion coefficient model, J. Mol. Liq. 243 (2017) 781-789. doi:10.1016/j.molliq.2017.08.069.

[24] Y. Hsu, Y. Chen, Correlation of the mutual diffusion coefficients of binary liquid mixtures, Fluid Phase Equilib. 152 (1998) 149-168.

[25] B.E. Poling, J.M. Prausnitz, J.P. O'Connell, The Properties of Gases and Liquids, Fifth, McGraw-Hill, New York, 2001.

[26] L.S. Darken, Diffusion, Mobility and Their Interrelation through Free Energy in Binary Metallic Systems, Trans. AIME. 175 (1948) 148-201.

[27] D.S. Abrams, J.M. Prausnitz, Statistical Thermodynamics of Liquid Mixtures : A New txpression for the Excess Gibbs Energy of Partly or Completely Miscible Systems, AIChE J. 21 (1975) 116-128.

[28] Z. Lei, J. Zhang, Q. Li, B. Chen, UNIFAC Model for Ionic Liquids, Ind. Eng. Chem. Res. 48 (2009) 2697-2704.

[29] E.L. Cussler, Cluster Diffusion in Liquids, AIChE J. 26 (1980) 43-51.

[30] M.B. Shiflett, M.A. Harmer, C.P. Junk, A. Yokozeki, Solubility and diffusivity of difluoromethane in room-temperature ionic liquids, J. Chem. Eng. Data. 51 (2006) 483-495. doi:10.1021/je050386z.

[31] A. Cera-Manjarres, Experimental determination and modelling of thermophysical properties of ammonia/ionic liquid mixtures for absorption refrigeration systems, Universitat Rovira i Virgili, 2015.

[32] K. Daucik, R.B. Dooley, Revised Supplementary Release on Properties of Liquid Water at 0.1 MPa, 2011.

[33] A. Mialdun, J.C. Legros, V. Yasnou, V. Sechenyh, V. Shevtsova, Contribution to the benchmark for ternary mixtures: Measurement of the Soret, diffusion and thermodiffusion coefficients in the ternary mixture THN/IBB/nC12 with 0.8/0.1/0.1 mass fractions in ground and orbital laboratories, Eur. Phys. J. E. 38 (2015). doi:10.1140/epje/i2015-15027-2.

[34] MATLAB and Statistics and Machine Learning Toolbox, Release 2018a, The MathWorks, Inc., Natick, Massachusetts, United Sates., (n.d.).

[35] C.-L. Wong, A.N. Soriano, M. Li, Diffusion coefficients and molar conductivities in aqueous solutions of 1-ethyl-3-methylimidazolium-based ionic liquids, Fluid Phase Equilib. 271 (2008) 43-52. doi:10.1016/j.fluid.2008.07.006.

[36] E.L. Cussler, Diffusion: Mass Transfer in Fluid Systems, 3rd ed., Cambridge University Press, 2009. 